\title{
The effects of wind and nonlinear damping on rogue waves and permanent downshift
}

\author{
C.M. Schober ${ }^{1}$ and M. Strawn \\ Department of Mathematics \\ University of Central Florida \\ Orlando, Florida, USA
}

\begin{abstract}
In this paper we investigate the effects of wind and nonlinear damping on permanent downshift and the formation of rogue waves in the framework of a HONLS model. Wind effects are incorporated by including a uniform linear damping/forcing term in the model. The strength of the wind, $\Gamma$, is allowed to vary as well as wind duration. Determining permanent downshift is not straightforward and we propose a criteria for permanent downshift related to our numerical experiments.

We consider large ensembles of initial data for modulated unstable Stokes waves with $N=1,2,3$ unstable modes. In the nonlinear damped HONLS evolution we find permanent downshift is observed whenever the strength of the nonlinear damping $\beta>0.1$. Notably, rogue waves typically do not develop after the time of permanent downshift, implying that a downshifted sea-state does not allow for any further rogue waves. Incorporating wind effects into the nonlinear damped HONLS model, we find that damping by the wind weakens downshifting while forcing by the wind enhances downshifting.

The proximity of the initial data to unstable plane waves impacts the characteristic features of the rogue waves in the nonlinear damped HONLS evolution. We find that as the initial data is chosen closer to the plane wave, the maximum strength, number, and lifetime of rogue waves increase while the time of permanent downshift decreases. Alternatively, we show that the greater the wave strength, the more rogue waves, or the longer their lifetime, the earlier permanent downshift occurs.

Keywords: Rogue wave, freak wave, downshifting, nonlinear damping, nonlinear Schrödinger equation, wind action.
\end{abstract}

\section{Introduction}

Associated with the Benjamin-Feir or modulational instability, where small perturbations of the Stokes wave lead to exponential growth of the sidebands, is a host of interesting phenomena, two of which are frequency downshifting and rogue waves. One of the simplest descriptions of the modulational instability

\footnotetext{
${ }^{1}$ Corresponding author's email: cschober@ucf.edu

Preprint submitted to PhysicaD
}

August 24, 2015

(C) 2015. This manuscript version is made available under the Elsevier user license http://www.elsevier.com/open-access/userlicense/1.0/ 
is provided by the nonlinear Schrödinger (NLS) equation which has been used as a first approximation for modeling rogue waves and addressing downshifting. Lake et. al. investigated analytically and experimentally the evolution of the modulational instability $[19,18]$. In their laboratory experiments, when the initial steepness of the waves was small, the wave trains experienced Fermi-Pasta-Ulam recurrence. On the other hand, when the wave steepness was large, the lower and upper sidebands grew at different rates and the energy permanently transferred from the carrier to a lower sideband, resulting in permanent frequency downshift. The corresponding numerical experiments using the conservative NLS equation showed a cyclical transfer of energy between the carrier and the sidebands, consistent with the observed recurrence for the waves with small steepness. Since the NLS equation preserves the symmetry of the Fourier components, it was not able to describe the downshift dynamics observed for waves with larger steepness.

The Dysthe equation, obtained by retaining terms through fourth order in an asymptotic expansion for the surface displacement, introduces asymmetry with respect to space translations [7]. The higher order corrections have a significant effect on the evolution of the modulational instability and it was conjectured that the Dysthe equation might capture the permanent downshift observed in the laboratory experiments. However, only temporary downshifting is obtained near the peaks of the modulation in numerical experiments [21]. Even though the momentum is not conserved by the Dysthe equation, there is insufficient change in the flux to allow for permanent downshift. Recently the Dysthe equation was brought into Hamiltonian form, providing a new higher order nonlinear Schrödinger (HONLS) equation [9]. Permanent downshift is not possible in the HONLS equation, even when steep waves are encountered, as the the energy and momentum, and thus the spectral center, are conserved.

Although a study of random waves with Gaussian spectrum yielded permanent downshift in an undamped Dysthe's equation [8], the general consensus is that damping plays an important role in downshifting. Recent studies on the effects of dissipation on the Benjaimin-Feir instability have shown numerically and in laboratory experiments that linear damping, even when weak, stabilizes the instability [26]. The exponential growth of the sidebands may no longer be saturated by the nonlinearity, rather the growth may be bounded by dissipation before the nonlinear terms become significant $[26,5]$. However, permanent downshift does not occur in higher order nonlinear Schrödinger models with only linear damping as the energy and momentum decay at the same rate which keeps the spectral center constant. Once nonlinear dissipation was included in Dysthe type models, permanent downshift was obtained for both breaking waves [27] and non-breaking waves $[10,15]$.

The perturbed Stokes wave initial data used in Lake's experiments are close to initial data for special solutions of the NLS equation: the multi-mode homoclinic orbits of unstable Stokes waves with N unstable modes (UMs). We refer to these homoclinic orbits, with $M \leq N$ modes excited, as the $M$ mode spatially periodic breather solutions (SPBs), which include the Akhmediev breathers when $N=1$. The SPBs capture the nonlinear stage of the modulational instability and are widely used to model rogue waves since they are 
transient, localized, and steep [1, 3, 24]. One and two mode SPBs have analytically been shown to persist in a conservative broadband HONLS equation [3]. In this paper we use modulated unstable Stokes waves initial data to simultaneously numerically investigate downshifting and rogue waves.

In our earlier study on rogue waves and downshifting using the new HONLS equation with nonlinear damping of the mean flow, the initial data were carefully chosen perturbations of unstable Stokes waves with three UMs, close to initial data for steep coalesced three-mode SPB solutions of the NLS equation. Due to the steepness of the waves, permanent downshift (here downshifting of the wavenumber is considered) occurred for all values of the nonlinear damping parameter $\beta$ considered, $0.05<\beta<0.75$ in the numerical experiments. Significantly, for Stokes waves with three UMs it was shown that rogue waves did not develop after the wavenumber permanently downshifted [14].

The nonlinear damping considered is such that steeper waves result in significantly stronger damping (see Figure 1). A natural question, which we address in this paper, is whether permanent downshift and the cessation of rogue waves after permanent downshift will be obtained when there are fewer unstable modes initially and the waves have smaller strength. Another novel aspect we investigate is how wind affects downshifting and rogue wave formation as well as the relation between the two phenomena. We conduct a broad numerical investigation of the effects of wind and nonlinear damping on permanent downshift and the formation of rogue waves in the framework of the HONLS model. Wind effects are incorporated by including a uniform linear damping/forcing term in the model. The strength of the wind, $\Gamma$, is allowed to vary as well as the duration of the wind, i.e. the wind acts for limited time $0 \leq t \leq T_{\text {wind }}$. Additional questions addressed in the current study are: i) whether the proximity of initial data to underlying instabilities of the plane wave affects rogue waves and downshifting and how to measure this and ii) how the strength of the waves affect the time of permanent downshift.

We consider large ensembles of initial data for modulated unstable Stokes waves with $N$ UMs (referred to as the $N$ UM regime). The initial data used in these experiments are more general than in the earlier study since the initial data are generic perturbations of Stokes waves which yield waves with smaller strength. Typically we examine the $N=1,2 \mathrm{UM}$ regime. For some of the issues not previously addressed, e.g. the combined effects of wind and nonlinear damping, the $N=3 \mathrm{UM}$ regime is also examined. We assume the waves do not break and that asymmetry develops on the same timescale as the action of the wind and nonlinear damping.

Section 2 provides background material: the properties of the governing equations, a linear stability analysis of Stokes waves for the new nonlinear damped HONLS, and the setup of the numerical experiments. As a point of comparison for the nonlinear damped HONLS evolution, we review the construction of rogue waves in the NLS equation and their appearance in the conservative HONLS equation (Section 3). Linear damping by the wind is examined in Section 4 which shows only temporary downshifting, as the theory predicts. In Section 5 nonlinear damping is introduced to the model and its effect on rogue waves and 
downshifting is quantified. Permanent downshift is observed, even for waves with smaller steepness, whenever the strength of the nonlinear damping $\beta>0.1$. Notably, rogue waves typically do not develop after the time of permanent downshift, implying that a downshifted sea-state does not allow for any further rogue waves. Additionally, we examine how the proximity of initial data to the plane wave shown impacts downshifting and the features of the rogue waves. The effect of wind and nonlinear damping on rogue waves and downshifting is considered in Section 6 where it is shown that damping by the wind weakens downshifting while forcing by the wind enhances downshifting.

\section{Analytical Background}

\subsection{Governing Equations}

Recently Gramstad and Trulsen brought the Dysthe equation, which approximately describes the evolution of slowly modulated periodic wave trains in deep water, into Hamiltonian form [9]. We add a linear damping/forcing term to the model to incorporate wind effects and a nonlinear damping term, the $\beta$-term, to model damping of the mean flow:

$$
i u_{t}+u_{x x}+2|u|^{2} u+i \Gamma u+i \epsilon\left(\frac{1}{2} u_{3 x}-8|u|^{2} u_{x}-2 u i(1+i \beta)\left[\mathcal{H}\left(|u|^{2} \mid\right)\right]_{x}\right)=0,
$$

where $u(x, t)$ is the complex envelope of a wavetrain, $\epsilon \geq 0$ and $\mathcal{H}(f(x))=\frac{1}{\pi} \int_{-\infty}^{\infty} \frac{f(\xi)}{x-\xi} d \xi$ is the Hilbert transform of $f$. Periodic boundary conditions, $u(x, t)=u(x+L, t)$, are considered. When $\beta=\Gamma=0$ equation (1) is referred to as the HONLS equation.

As can be deduced from equation (3), nonlinear damping requires $\beta>0$. Figure 1 shows $|u(x, t)|$ (solid line), and the corresponding $\beta$-term $\left|u\left[\mathcal{H}\left(|u|^{2} \mid\right)\right]_{x}\right|$ (dashed line), when the wave train is strongly modulated at $t=t^{*}$. The $\beta$ term is large only near the two steep crests at $x \approx-7$ and $x \approx 2.5$ while the $\beta$ term is nearly negligible for the background state. As a result, the effects of the nonlinear damping are localized in space time where $u(x, t)$ is steep, with steeper waves producing stronger damping. This is important to bear in mind since one of our primary questions is how nonlinear damping affects rogue waves. When $\beta>0$ and $\Gamma=0$, equation (1) is referred to as the nonlinear damped HONLS equation.

The effects of wind on the waves can be incorporated into the system with the inclusion of the linear $\Gamma$ term $[20,17]$. When $\Gamma>0$ the wind opposes the wave evolution, causing surface damping. When $\Gamma<0$ the wind acts in the direction of the wave evolution and forces the waves. For brevity, when nonlinear damping $\beta>0$ and wind $\Gamma \neq 0$ are present, equation (1) is referred to as the perturbed HONLS equation. The wind is considered to act for limited time $0 \leq t \leq T_{\text {wind }}$. In formulations of the HONLS, where space and time are interchanged, varying $T_{w i n d}$ is equivalent to varying the fetch. 


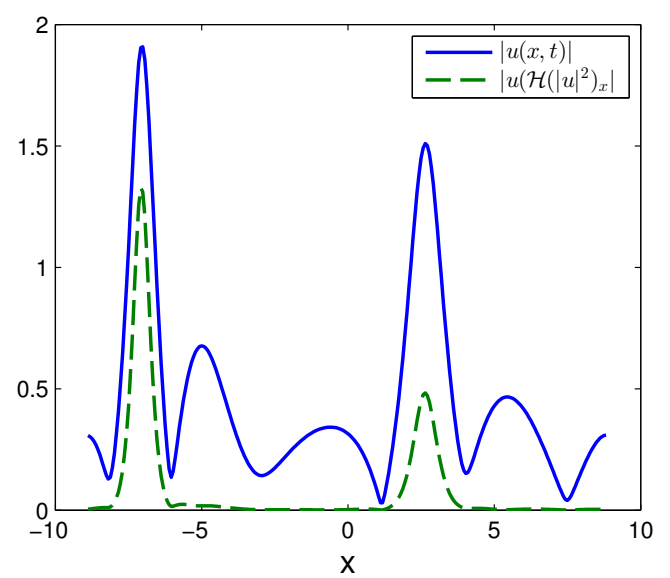

Figure 1: Solution of the nonlinear damped HONLS, $\epsilon=0.05, \beta=0.1, \Gamma=0$, at $t^{*}=27.6$ for $u(x, 0)=0.7(1+0.1 \cos \mu x)$ with $L=4 \sqrt{2} \pi$.

\subsection{Wave energy and Flux}

The total wave energy, $E$, and the momentum or total energy flux, $P$ are defined as

$$
E(t)=\int_{0}^{L}|u|^{2} \mathrm{~d} x \quad \text { and } \quad P(t)=i \int_{0}^{L}\left(u^{*} u_{x}-u u_{x}^{*}\right) \mathrm{d} x .
$$

To determine how the perturbation affects the wave energy, we examine the rate of change

$$
\frac{d E}{d t}=-2 \int_{0}^{L}|u|^{2}\left(\Gamma+2 \epsilon \beta\left[\mathcal{H}\left(|u|^{2}\right)\right]_{x}\right) d x
$$

Note the wave energy is conserved for both the NLS and the HONLS equations $(\Gamma=\beta=0)$. To see more clearly the dependence of the wave energy on $\beta$ and $\Gamma$, expand $u(x, t)$ and $|u(x, t)|^{2}$ in the following Fourier series,

$$
u(x, t)=\sum_{k=-\infty}^{\infty} \hat{u}_{k}(t) e^{i k x} \quad|u(x, t)|^{2}=\sum_{k=0}^{\infty}\left(\alpha_{k}(t) e^{i k x}+\alpha_{k}^{*}(t) e^{-i k x}\right)
$$

Substituting these expansions into equation (2), one arrives at the Fourier expansion for $\frac{d E}{d t}$,

$$
\frac{d E}{d t}=-2 L\left[\Gamma \sum_{k=-\infty}^{\infty}\left|\hat{u}_{k}\right|^{2}+2 \epsilon \beta \sum_{k=1}^{\infty} k\left|\alpha_{k}\right|^{2}\right]
$$

Clearly $\beta>0, \Gamma=0$ corresponds to nonlinear damping. For $\beta>0$ : 1) If $\Gamma>0$, then $\frac{d E}{d t}<0$ for all time and the total energy is dissipated. 2) If the wind is pumping energy into the system, $\Gamma<0$, then for a given $\beta$ the total energy can transition in time between being damped and being forced. In this paper we only consider damped forced regimes, i.e. $(\beta, \Gamma)$ pairs, where damping controls the growth from the wind on the time frame examined. See section 2.6 for further details. 
The evolution of the momentum for the perturbed HONLS equation is given by

$$
\frac{d P}{d t}=-2 i \int_{0}^{L}\left(u^{*} u_{x}-u u_{x}^{*}\right)\left[\Gamma+2 \epsilon \beta\left(\mathcal{H}\left(|u|^{2}\right)\right)_{x}\right] d x .
$$

Again, since $\Gamma=\beta=0$, the momentum is conserved for the NLS and HONLS equations. The Fourier expansion of the momentum can be written as

$$
P=-2 L \sum_{-\infty}^{\infty} k\left|\hat{u}_{k}\right|^{2}=2 L \sum_{k=1}^{\infty} k\left(\left|\hat{u}_{-k}\right|^{2}-\left|\hat{u}_{k}\right|^{2}\right)
$$

which measures the asymmetry of the Fourier modes. This suggests that the momentum will be important in understanding downshift in the spectrum.

The Hamiltonian for the HONLS equation is given by

$$
\mathcal{H}=\int_{0}^{L}\left\{-i\left|u_{x}\right|^{2}+i|u|^{4}-\frac{\epsilon}{4}\left(u_{x} u_{x x}^{*}-u_{x}^{*} u_{x x}\right)+2 \epsilon|u|^{2}\left(u^{*} u_{x}-u u_{x}^{*}\right)+i \epsilon|u|^{2}\left[H\left(|u|^{2}\right)\right]_{x}\right\} d x .
$$

For the diagnostics related to downshifting we monitor the wave energy and momentum. The Hamiltonian for the HONLS equation provides a third conserved quantity with which to monitor the accuracy of the numerical experiments.

\subsection{Measuring downshifting: the spectral peak and spectral center}

The modulational instability is at the heart of the downshifting phenomenon. Within the conservative NLS and HONLS models, when the Stokes wave is modulated the sidebands grow due to an energy transfer from the carrier mode to a sideband which then becomes dominant. This transfer of energy can be thought of as a temporary downshift as the wavetrain undergoes successive modulations and demodulations (energy is transferred back and forth between the carrier and sideband modes) until the original state reoccurs with

the carrier wave dominant. However, in the laboratory experiments of Lake et. al., when the waves are steep enough, the transfer of energy to a lower sideband can become permanent [19]. This phenomenon is not described by the NLS or HONLS equations.

Two different quantities, the spectral peak and the spectral center, have been developed to examine downshifting in a sea state. Defining the dominant mode or spectral peak, $k_{\text {peak }}$, as the wave number $k$ for which $\left|\hat{u}_{k}\right|$ achieves its maximum, downshifting occurs when there is a shift down in $k_{\text {peak }}$ [28]. One may also consider the center, $k_{m}$, of the spectrum. In analogy with the analysis of $u(x, t)$ on an infinite domain where Parseval's theorem and the generalized mean value theorem yield the existence of a spectral mean $k^{*}=-\frac{1}{2} \frac{P}{E}$, Uchiyama and Kawahara defined, for the periodic domain, the spectral center or mean wave number as [29]:

$$
k_{m}=-\frac{1}{2} \frac{P}{E}=\frac{\sum_{-\infty}^{\infty} k\left|\hat{u}_{k}\right|^{2}}{\sum_{-\infty}^{\infty}\left|\hat{u}_{k}\right|^{2}} .
$$


Using the spectral center, downshifting is considered to occur when $k_{m}$ is generally decreasing, i.e. $\dot{k}_{m}<0$.

Permanent downshift from the carrier wave is considered to occur when the spectral peak $k_{\text {peak }}$ moves permanently to a lower mode [28]. The time of permanent downshift is defined to be the last time the original carrier wave is the dominant mode. Although other studies obtained consistent results on downshifting when using $k_{\text {peak }}$ and $k_{m}$ [12], in our numerical studies there are several experiments where even though $\dot{k}_{m}<0$, $k_{\text {peak }}$ does not permanently downshift on the time frame under study. We arrive at the following conjecture related to our numerical experiments (see Figure 13 and a discusion of the initial data at the end of Section 5.3):

Conjecture 1. It appears that rapid, generally increasing/decreasing, step-like changes in the flux/energy with $|\dot{P}|>|\dot{E}|$ are essential for permanent downshift.

Further, the evolution of $k_{m}$ does not provide precise information on the time of permanent downshift. As a result, using $k_{m}$ in conjunction with $k_{\text {peak }}$ provides a more complete description of the spectral activity: we use the spectral center to provide a necessary condition for permanent downshift, i.e. $k_{m}$ is generally decreasing, and we use $k_{\text {peak }}$ to determine the time at which the downshift becomes permanent.

For both the NLS and HONLS equations $k_{m}$ is constant since the energy and momentum are conserved. Although temporary downshifts can occur in the NLS and HONLS numerical experiments (see Section 3 ), a permanent downshift does not occur since $k_{m}$ is constant. When $\Gamma \neq 0$ and $\beta=0$ one finds from equations (2) and (4) that the energy and the momentum decay at the same rate, $E(t)=E(0) \exp (-2 \Gamma t)$ and $P(t)=P(0) \exp (-2 \Gamma t)$. As a result, for the linear damped/forced HONLS equation we do not expect a permanent downshift in the spectrum since the spectral center $k_{m}$ is constant in time.

Finally, in some of the experiments we observe continued spectral activity in the lower modes after permanent downshift, especially when both wind and nonlinear damping are active. In this case, when addressing questions related to the time of the last rogue wave, it becomes relevant to also consider the time when $k_{\text {peak }}$ settles at it's final wavenumber, $k_{\text {peak }}^{\text {final }}$.

\subsection{Linear Instability of the Stokes wave for the HONLS and nonlinear damped HONLS equations}

The NLS, HONLS and nonlinear damped HONLS equations, i.e. equation (1) with $\epsilon \geq 0, \beta \geq 0$, and $\Gamma=0$, all admit plane wave or Stokes wave solutions,

$$
u_{a}(t)=a \mathrm{e}^{2 i a^{2} t} .
$$

We take $a \in R$ since the equation is invariant under the transformation $a \rightarrow a e^{i b}$. The stability of these solutions with respect to small $(\hat{\epsilon}<<1)$ perturbations in amplitude and phase can be examined by assuming

$$
u(x, t)=u_{a}(t)\left(1+\hat{\epsilon}\left(A_{1} \phi+A_{2} \phi^{*}\right)\right)
$$


where $A_{1}, A_{2} \in \mathbb{R}$ and $\phi=\exp (i k x-i \Omega t)$. The wavenumber $k$ is real but $\Omega$ may be complex. Substituting the perturbed plane wave assumption (8) into equation (1) with $\Gamma=0$ and linearizing yields the following equation for $A_{1}$ and $A_{2}$,

$$
A_{1}\left[(\Omega+C-B) \phi+(D-B) \phi^{*}\right]+A_{2}\left[\left(-\Omega^{*}+E-B\right) \phi^{*}+(D-B) \phi\right]=0,
$$

where

$$
\begin{aligned}
& B=2 i a^{2} k \epsilon \beta \\
& C=-k^{2}+2 a^{2}+\frac{1}{2} \epsilon k^{3}+10 a^{2} \epsilon k \\
& D=2 a^{2}+2 a^{2} k \epsilon \\
& E=-k^{2}+2 a^{2}-\frac{1}{2} \epsilon k^{3}-6 a^{2} \epsilon k
\end{aligned}
$$

Forming the conjugate equation and requiring the determinant of the coefficient matrix for these two equations to be zero gives our dispersion relation:

$$
\Omega=\epsilon\left(-\frac{1}{2} k^{3}-8 a^{2} k-2 i a^{2} k \beta\right) \pm i k \sqrt{4 a^{2}-k^{2}+4 a^{4} \epsilon^{2} \beta^{2}+4 a^{2} k \epsilon}
$$

The growth rates of the sidebands are given by the $\operatorname{Im}(\Omega)$,

$$
\sigma_{j}=\operatorname{Im}(\Omega)=-\epsilon 2 a^{2} k_{j} \beta \pm k_{j} \sqrt{4 a^{2}-k_{j}^{2}+4 \epsilon a^{2} k_{j}+4 \epsilon^{2} a^{4} \beta^{2}},
$$

where $k_{j}=2 \pi j / L$, and are shown in Figure 2. When $\epsilon=0$ we recover the growth rates for the NLS equation

$$
\sigma_{j}= \pm k_{j} \sqrt{4 a^{2}-k_{j}^{2}}
$$

The cutoff wavenumber for unstable modes, $K^{*}$, occurs when the growth rate $\sigma=0$, e.g. $\left(K^{*}\right)^{2}=4 a^{2}$ for the NLS. As a result the plane wave is unstable with respect to long wavelength perturbations, i.e. if

$$
0<\left(\frac{\pi j}{L}\right)^{2}<a^{2}, \quad j \in \mathbb{N},
$$

then $u_{a}$ has $N=\lfloor a L / \pi\rfloor$ unstable modes. For the HONLS and nonlinear damped HONLS equations, $K^{*}=2 a \sqrt{1+\epsilon^{2} a^{2}}-2 \epsilon a^{2}$, which is close to that for the NLS equation (see Figure 2). The growth rates satisfy the inequality: $\sigma_{N L S}<\sigma_{\text {nldampedHONLS }}<\sigma_{H O N L S}$. For small $\epsilon, \beta<<1$ the growth rate of the most unstable mode is only slightly modified and the initial stage of the modulation will not change significantly. Throughout this paper the $\mathrm{N}$ unstable mode "regime" refers to the range in the Stokes wave parameters $a$ and $L$ which allow $N$ unstable modes initially.

\subsection{Rogue waves in the NLS framework}

The evolution of the strength of a wave is given by

$$
S(t)=\frac{U_{\max }(t)}{H_{s}(t)}
$$




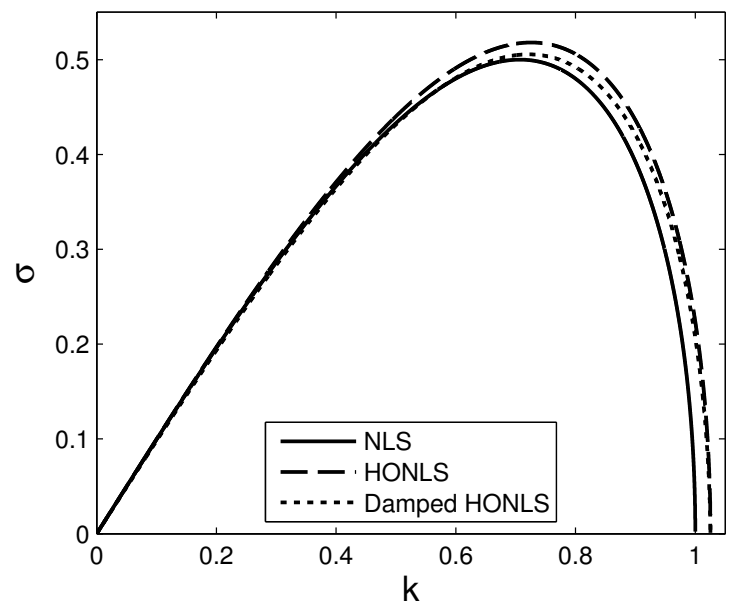

Figure 2: Growth rates, $\sigma_{j}$, of the sidebands for the NLS $(\epsilon=\beta=0)$, HONLS $(\epsilon=0.05, \beta=0)$, and nonlinear damped HONLS $(\epsilon=0.05, \beta=.7)$ equations as a function of $k_{j}$ for $a=.5, L=4 \sqrt{2} \pi$.

where $U_{\max }(t)=\max _{x \in[0, L]}|u(x, t)|$ and $H_{s}(t)$ is the significant wave amplitude, defined as 4 times the standard deviation of the surface amplitude. A rogue wave is considered to occur at time $t^{*}$ if $S\left(t^{*}\right)>2.2$. For a particular time series the maximum strength is defined as $S_{\max }=\max _{t \in[0, T]} S(t)$ and the lifetime of rogue events is defined to be the total length of time that $S(t)>2.2$. For a family of waves depending on a parameter $\alpha$, the dependence of the strength on $\alpha$ is indicated by $S(t ; \alpha)$ with corresponding $S_{\max }(\alpha)$.

Breather type solutions of the NLS equation have been widely used as models for rogue waves as they are transient, localized and steep [4]. Explicit formulas for M-mode spatially periodic breathers (SPBs) over an unstable plane wave with $\mathrm{N}$ unstable modes $(N \geq M)$ can be obtained using the Bäcklund-gauge transformation for the NLS equation [25]. An important feature of the SPBs is their localization in time: as $t \rightarrow \pm \infty$ the breather exponentially decays to a phase translate of the original Stokes wave at the rate $\sigma_{j}$. The SPBs are periodic in space and when the period $L$ is large they are also localized in space.

\subsubsection{Single mode spatially periodic breathers}

The $N$ unstable modes of the unstable plane wave "seed" solution have $N$ corresponding degenerate complex discrete spectra $\lambda_{j}^{d}$ of the associated Zakharov-Shabaat linear system. A single Bäcklund transformation at $\lambda_{j}^{d}$ generates a single mode SPB corresponding to the $\mathrm{j}$-th unstable mode. The formula for the single mode SPB associated with the $j$-th $\mathrm{UM}$ of $u_{a}$ (for $1 \leq j<a L / \pi$ ) is

$$
U^{(j)}(x, t)=a \mathrm{e}^{\mathrm{i}\left(2 a^{2} t+\phi\right)} \frac{\cos 2 p_{j}-\sin p_{j} \operatorname{sech}\left(\rho-\sigma_{j} t\right) \cos (2 \pi j x / L+\alpha)+\mathrm{i} \sin 2 p_{j} \tanh \left(\rho-\sigma_{j} t\right)}{1+\sin p_{j} \operatorname{sech}\left(\rho-\sigma_{j} t\right) \cos (2 \pi j x / L+\alpha)},
$$

where $\cos p_{j}=\pi j / a L$ and $\rho$ and $\alpha$ are real temporal and spatial phase parameters. Figure 3 shows the amplitude of the single mode SPB, $U^{(1)}(x, t ; \rho)$, over the plane wave with one UM for $a=.5$ and $L=2 \sqrt{2 \pi}$. 


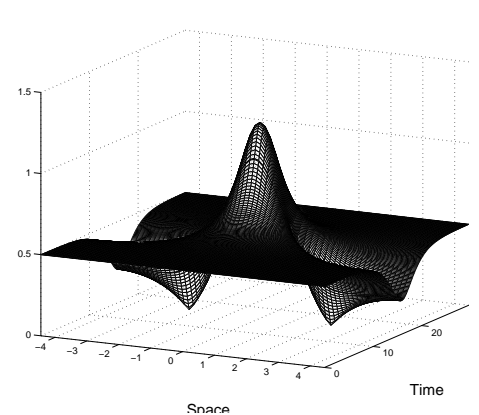

(a)

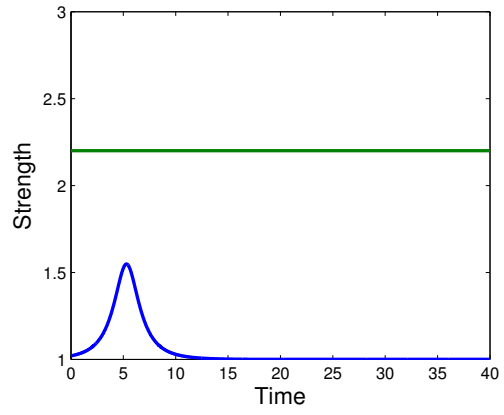

(b)

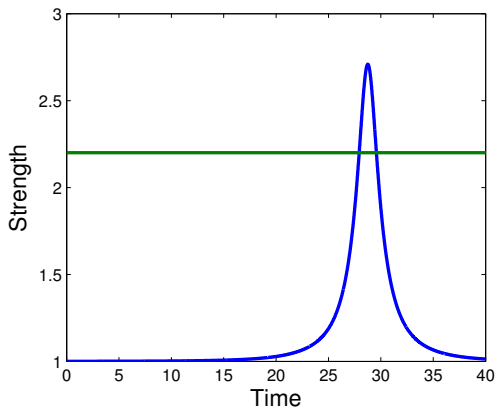

(c)

Figure 3: (a) Amplitude of the single mode SPB $U^{(1)}(x, t ; \rho)$ over an unstable plane wave with one UM: $a=0.5, L=2 \sqrt{2} \pi$. Strength of $\left|U^{(1)}(x, t ;-3)\right|$ with $a=.5$ for (b) $L=2 \sqrt{2} \pi$; (c) $a=.5, L=60$.

The amplification factor of the amplitude for $U^{(1)}(x, t ; \rho)$ is given by

$$
A f=\frac{\max _{x \in[0, L], t \in \mathbb{R}}|u(x, t)|}{\lim _{t \rightarrow \pm \infty}|u(x, t)|} \approx 2.4 .
$$

As $L$ increases the underlying plane wave has $N \geq 1 \mathrm{UMs}$; even so $U^{(1)}(x, t ; \rho)$ retains qualitatively the same structure as seen in Figure 3(a) over the larger period. Given that $A f$ depends upon the wavenumber of the modulation, as $\mathrm{L}$ increases the wave number decreases and the amplification factor increases to the limiting value $A f_{\text {max }}=\lim _{\kappa \rightarrow 0^{+}} A f(\kappa) \approx 3.1$.

Care should be exercised when using the one mode SPB as a model for rogue waves. When $U^{(1)}(x, t ; \rho)$ is over a plane wave with one UM it does not satisfy the strength definition of a rogue wave. The strength of the SPBs also depends upon the period $L$; we indicate this by $S(t ; L)$. We can characterize the one UM regime of the plane wave by fixing $a=.5$ and letting $2 \pi<L<4 \pi$. The strength $S(t ; L)$ of $U^{(1)}(x, t ; \rho)$ is an increasing function of $L$ and in the one UM regime the $\max _{L} \max _{t} S(t ; L)=1.98$ when $L=4 \pi$. In fact, for $a=0.5$, $U^{(1)}(x, t ; \rho)$ first qualifies as a rogue wave, $\max _{t} S(t ; L)=2.21$, when $L=L^{*} \approx 20$ and the underlying plane wave has $\mathrm{N}=3$ UMs. $U^{(1)}(x, t ; \rho)$ is $\epsilon$ close to it's maximum strength, $\max _{L} \max _{t} S(t ; L) \approx 2.7$, when $L \approx 60$. This limiting behavior is illustrated in Figures 3(b-c) which shows the wave strength for $U^{(1)}(x, t ; \rho)$ with $a=0.5$ for (b) $L=2 \sqrt{2} \pi$ and (c) $L=60$.

\subsubsection{Two mode spatially periodic breathers}

Higher dimensional M mode SPBs $(2 \leq M \leq N)$ are generated by iterating the Bäcklund transformation $M$ times. Each iteration introduces an additional parameter in the solution. For example, applying the Bäcklund-gauge transformation successively at complex $\lambda_{1}^{d}$ and $\lambda_{2}^{d}$ produces a two-mode SPB family of the form (see [22] for the exact formula):

$$
U^{(1,2)}(x, t ; \rho, \tau)=a e^{2 i a^{2} t} \frac{N(x, t ; \rho, \tau)}{D(x, t ; \rho, \tau)} .
$$




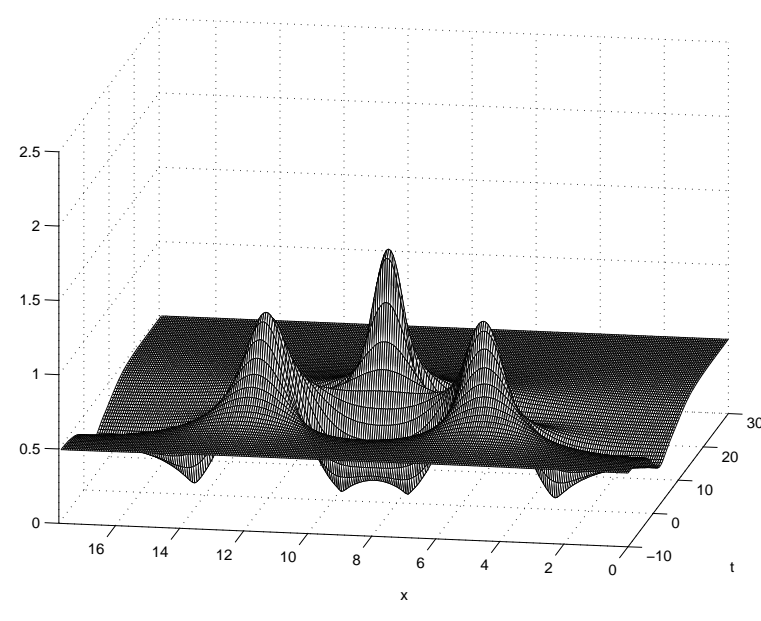

(a)

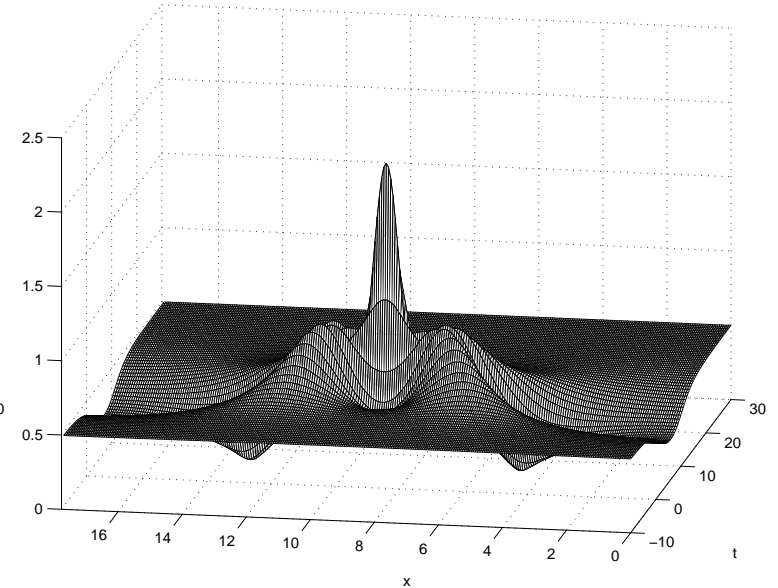

(b)

Figure 4: The amplitude of $U^{(1,2)}(x, t ;-2, \tau)$ with $a=.5, L=4 \sqrt{2} \pi$, where (a) the modes are well separated $(\tau=-2)$, and (b) the modes are coalesced $(\tau=-3)$.

The parameters $\rho$ and $\tau$ determine the time at which the first and second mode, respectively, are excited and as a result govern the shape and steepness of the SPB. Figure 4 shows the amplitude of $U^{(1,2)}(x, t ;-2, \tau)$ with $a=0.5$ and $L=4 \sqrt{2} \pi$ (the underlying plane wave has two UMs) when (a) the two spatial modes are well separated, $\tau=-2$, and (b) the two modes are coalesced, $\tau=-3$.

The two mode SPB qualifies as a rogue wave for all values of $\tau$ when $L=4 \sqrt{2} \pi$ and $a=.5$ since the maximum strength of $U^{(1,2)}(x, t ;-2, \tau)$ ranges from $\max _{t} S(t) \approx 2.21$ when the modes are well separated to $\max _{t} S(t) \approx 3.46$ when the modes are coalesced, see Figure 5 (solid line). However, very close to the lower threshold for two UMs, e.g. $a=.5$ and $L=4.04 \pi, U^{(1,2)}(x, t ;-2, \tau)$ may not satisfy the strength definition of a rogue wave, see Figure 5 (dashed line). From the perspective of the family of two mode SPBs, for a given $\mathrm{L}$ and amplitude, there exists $\tau_{1}<\tau<\tau_{2}$ for which $U^{(1,2)}(x, t ;-2, \tau)$ is a rogue wave.

\subsection{Setup of numerical experiments}

We consider initial data of the form used in Lake's experiments [19], i.e. small perturbations of unstable plane waves,

$$
u(x, 0)=a(1+\delta \cos 2 \pi x / L),
$$

where $\delta<<1$ and the amplitude $a$ and period $L$ are chosen so that $\lfloor a L / \pi\rfloor=N=1, \ldots, 3$ (referred to as the N unstable mode (UM) "regime"). The initial data is also close to that of the SPBs of the NLS discussed in section 2.5. Rogue waves do not occur in the one UM regime, typified by waves with smaller strength and steepness. The $N \geq 2 \mathrm{UM}$ regime is effectively a rogue wave regime for the HONLS equation. It should be noted that the initial data used in these experiments is more general than in the earlier study 


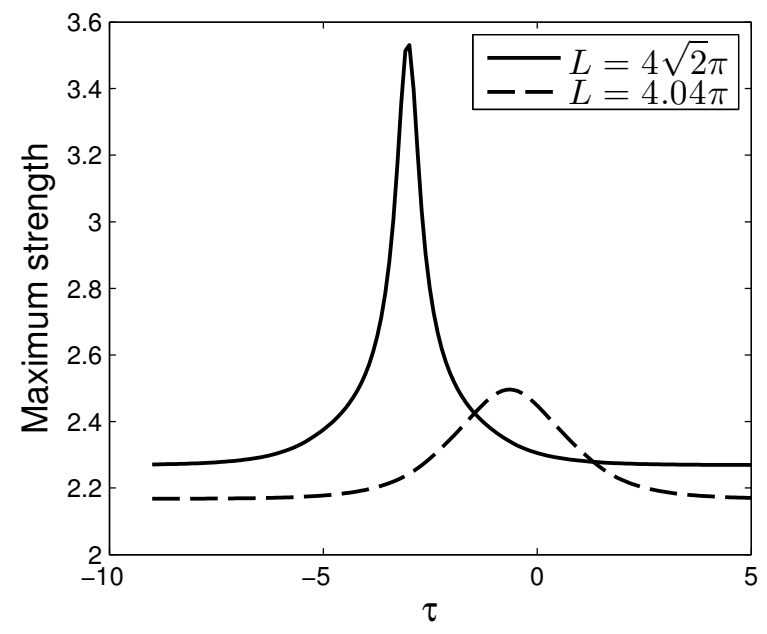

Figure 5: Plots of the maximum strength of $U^{(1,2)}(x, t ;-2, \tau)$, as a function of $\tau$ with $a=.5, L=4 \sqrt{2} \pi$ (solid line), and $L=4(1+.01) \pi$ (dashed line).

focusing on the three unstable mode regime [14] since aside from having fewer unstable modes, the initial data are generic perturbations of the plane wave, rather than carefully selected initial data for a very steep coalesced solution.

The governing equation (1) is solved numerically using a very accurate smoothing exponential integrator. The integrator combines a Fourier-mode decomposition in space with a fourth-order exponential RungeKutta method in time which uses Padé approximations of the matrix exponential terms [6, 16]. For a given accuracy, this exponential integrator was found to be up to an order of magnitude more efficient than standard pseudospectral and Fourier split-step methods in solving the NLS and HONLS equations [11].

The number of Fourier modes and the time step used in the experiments depends on the complexity of the solution. For example, for initial data in the two UM regime, typically $L=4 \sqrt{2} \pi$ and $N=256$ Fourier modes are used with time step $\Delta t=10^{-3}$. This space - time resolution allows for the three global invariants of the conservative HONLS equation, the energy $E$, momentum $P$, and Hamiltonian $\mathcal{H}$, to be conserved with an accuracy of $\mathcal{O}\left(10^{-12}\right), \mathcal{O}\left(10^{-11}\right), \mathcal{O}\left(10^{-11}\right)$, respectively for $0 \leq t \leq 200$. For the linear perturbed HONLS, $k_{m}=\frac{-P}{2 E}$ is invariant and, in the case of linear damping, is conserved with an accuracy of at least $\mathcal{O}\left(10^{-11}\right)$ for experiments in the $N=1,2,3$ UM regimes for $0 \leq t \leq 200$.

Typically the experiments are presented over the timeframe $0 \leq t \leq 200$ or $0 \leq t \leq 400$. However, key experiments, e.g. those relating the time of permanent downshift and the time of the last rogue wave, are run at least 50 units further in time to ensure $k_{\text {peak }}$ achieves $k_{\text {peak }}^{\text {final }}$. To avoid aliasing we eliminate the high frequency modes at every time step, e.g. when $N=256$ Fourier modes are used we let $\hat{u}_{k}=0$ for $|k|>120$. This does not have a significant effect on the exchange of energy between the dominant low wave number modes and thus does not change our results related to downshifting and rogue waves (it was also verified 


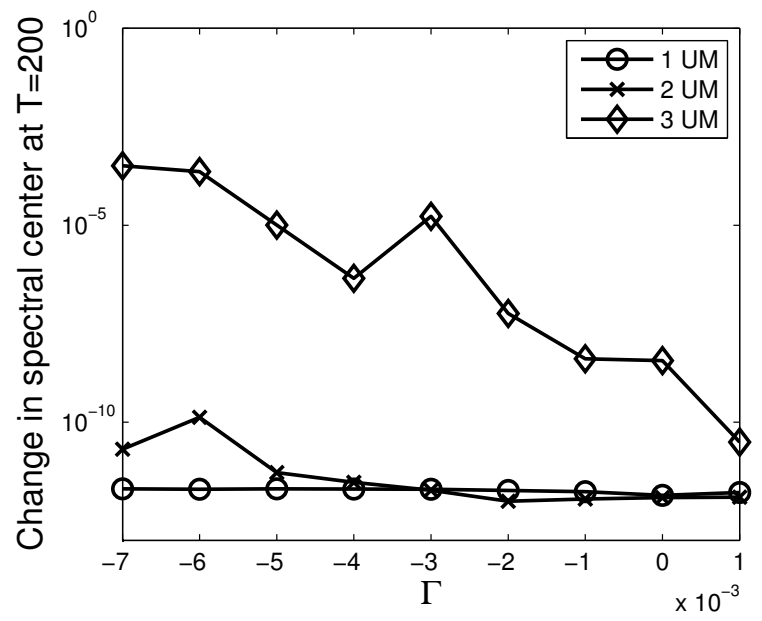

Figure 6: The averaged error of the terminal spectral center in the $N=1,2,3$ UM regimes for the linear perturbed HONLS, $\epsilon=0.05, \beta=0,-0.007 \leq \Gamma \leq 0.001$, for $0 \leq t \leq 200$. The wind acts until $T_{\text {wind }}=25$.

that varying the number of frequencies being eliminated does not change the behavior). Additionally, the behavior of the solution and of the dominant modes does not change when the mesh is refined.

The spectral center, $E, P$, and $\mathcal{H}$ are not invariant for the perturbed HONLS equation with nonlinear damping and linear forcing. As a result, we appeal to error results for the linear forced HONLS equation to provide an upper bound on the error in the numerical experiments which also include nonlinear damping. To be conservative, when both nonlinear damping and linear forcing are present we use the space-time mesh and parameter range in $\Gamma$ that allows for an acceptable conservation in the spectral center for the linear forced HONLS equation.

To determine the accuracy in the linear forced HONLS experiments for $0 \leq t \leq 200$ with wind acting until $T_{\text {wind }}=25$, for each of nine values of $\Gamma,-0.007 \leq \Gamma \leq 0.001$, five experiments using different amplitudes in the initial data were carried out in each of the $N=1,2,3 \mathrm{UM}$ regimes, (a) $.25 \leq a \leq .33$, (b) $.4 \leq a \leq .48$, (c) $.57 \leq a \leq .65$, respectively. For each value of $\Gamma$, the error in the spectral center, $k_{m}(200)-k_{m}(0)$, is averaged over the five experiments in each of the regimes. Figure 6 shows that for $T_{\text {wind }}=25$, the maximum error in the spectral center at $T=200$ is $\mathcal{O}\left(10^{-12}\right), \mathcal{O}\left(10^{-10}\right)$, and $\mathcal{O}\left(10^{-4}\right)$ for initial data in the $\mathrm{N}=1,2,3$ UM regimes, respectively. The error in the spectral center decreases as $T_{\text {wind }}$ decreases (not shown).

\section{Rogue waves and temporary downshifting in the HONLS equation}

In this section we compare the development of rogue waves and downshifting in the NLS and HONLS equations. We begin with modulated plane wave initial data (16) in the one UM regime, which can be characterized by fixing $L=2 \sqrt{2} \pi$ and varying the amplitude, $0.4 \leq a \leq .67$, with modulation $\delta=.0001$. Figure 7(a) shows the maximum strength, $S_{\max }(a)$, attained with the NLS and HONLS $(\epsilon=0.05)$ equations 
for $0 \leq t \leq 200$. For all values of $a$, the maximum strengths of the HONLS waves are greater than those of the NLS waves. Even so, whether using modulated plane wave or single mode SPB initial data, Figures 7(a) and (b) respectively, show rogue waves are not obtained in either the NLS or the HONLS experiments in the one UM regime.

In general, $S_{\max }$ increases with the initial amplitude; close to the upper bound for one UM $S_{\max }(.67) \approx$ 2.15 for the HONLS equation. As such, it may be possible to obtain rogue waves in the HONLS equation for initial data in the one UM regime by using larger values of $\epsilon$ and thereby introducing a second UM. Precisely becasue of this issue, we fix $\epsilon=0.05$ in our numerical experiments (which is at the lower range of values used by Lake et. al.). We are interested in studying the effect of nonlinear damping on downshifting and the SPBs in the $N=1,2,3 \mathrm{UM}$ regimes (as the number of unstable modes increases the amplitudes achievable by the rogue waves increase). The numerical experiments are also monitored by calculating the nonlinear mode content (not shown) from the inverse spectral theory of NLS of the waves and for larger values of $\epsilon$ more modes become active before nonlinear damping becomes significant. This clouds the issue of the effect of nonlinear damping and downshifting on an N-mode rogue wave.

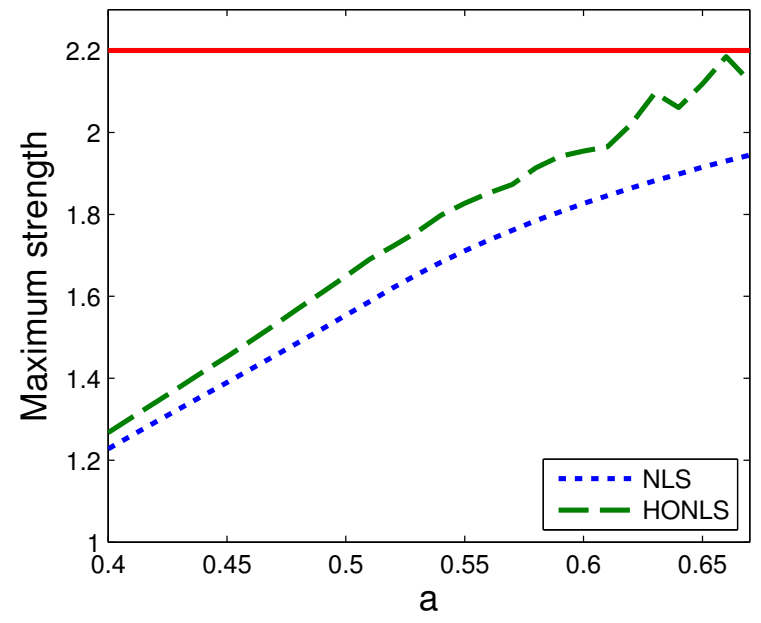

(a)

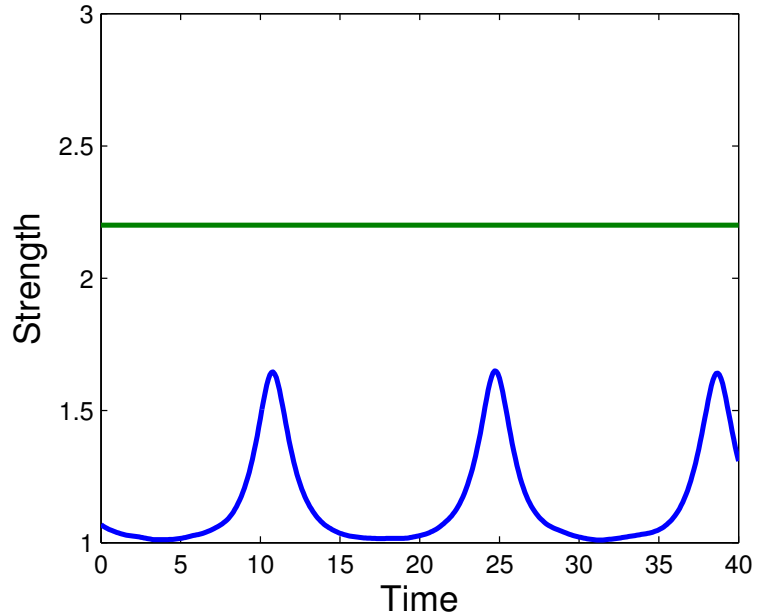

(b)

Figure 7: (a) $S_{\max }(a)$ for the NLS $(\epsilon=0)$ and HONLS $(\epsilon=0.05)$ equations using initial data (16) with $\delta=.0001$ and $L=2 \sqrt{2} \pi$, for $0 \leq t \leq 200$; (b) HONLS evolution of the single mode SPB initial data, $U^{1}(x, 0 ;-2), L=2 \sqrt{2} \pi, \epsilon=0.05$, $\beta=\Gamma=0$, for $0 \leq t \leq 40$.

For the HONLS equation, Figure 8(a) shows the surface amplitude $|u(x, t)|$ using initial data (16) in the two UM regime, i.e. $a=0.5, \delta=0.1$, and $L=4 \sqrt{2} \pi$ for $0 \leq t \leq 200$. Shortly after a rogue waves appear at $t \approx 8$ the waveform becomes irregular with two modes switching intermittently between traveling to the left and traveling to the right. The evolution of the strength $S(t)$, Figure $8(\mathrm{~b})$, shows rogue waves occur intermittently throughout the time series. The larger rogue waves have strengths $S(t)>2.6$ at $t \approx 8$ and at 
$t \approx 173$ which are due to partial coalescence of the modes in the chaotic state and which are greater than the strengths of the well separated two mode SPBs (see Figure 5). However, $S_{\max }$ for the HONLS equation are smaller than $S_{\max }$ for the coalesced two mode SPB as the higher order terms in the HONLS equation break symmetry and prevent a complete spatial coalescence of the nonlinear modes.

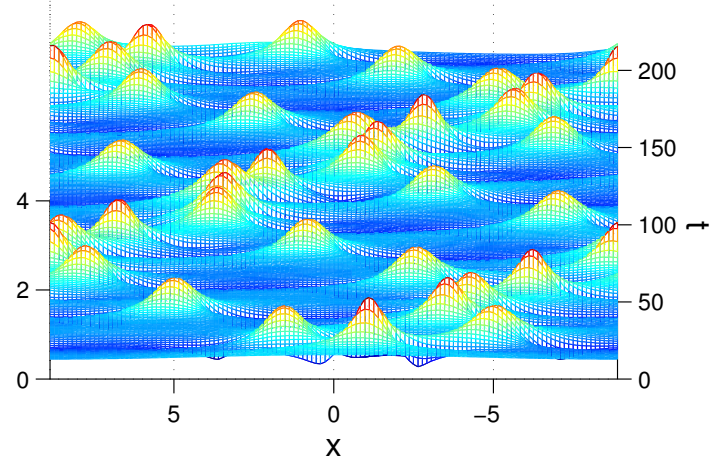

(a)

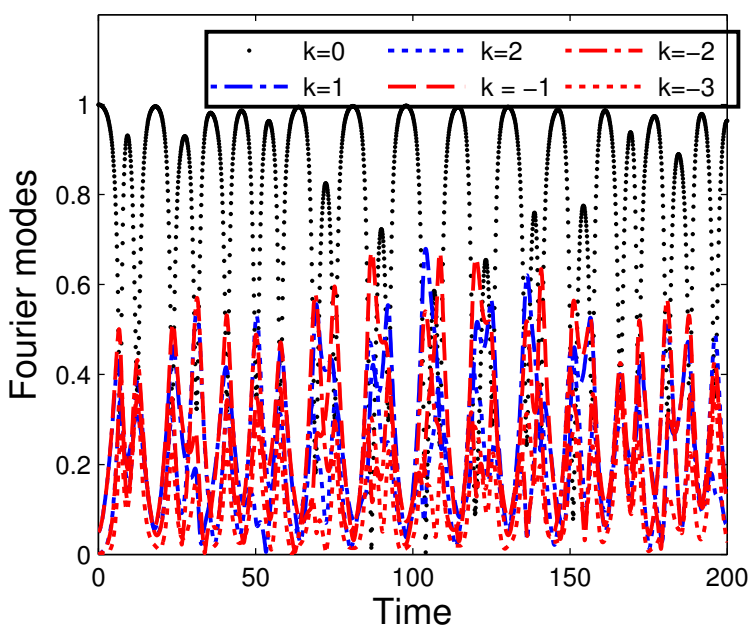

(c)

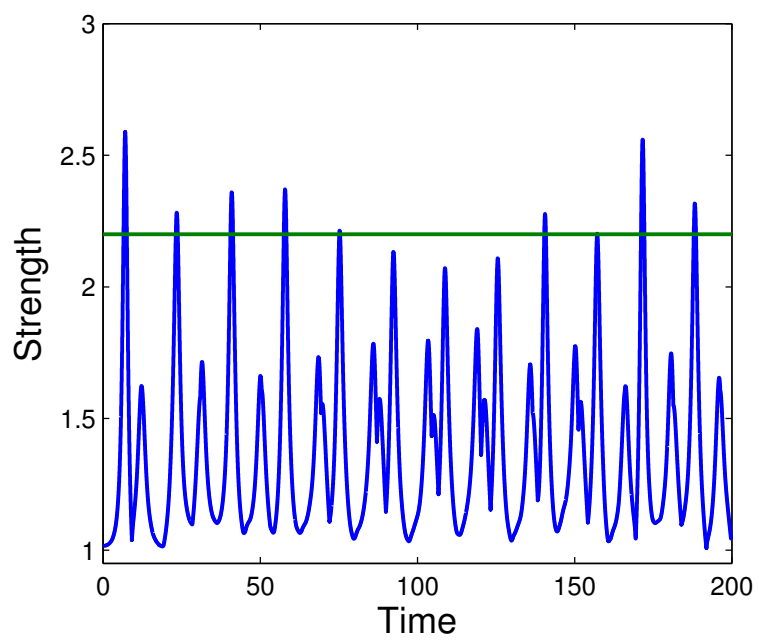

(b)

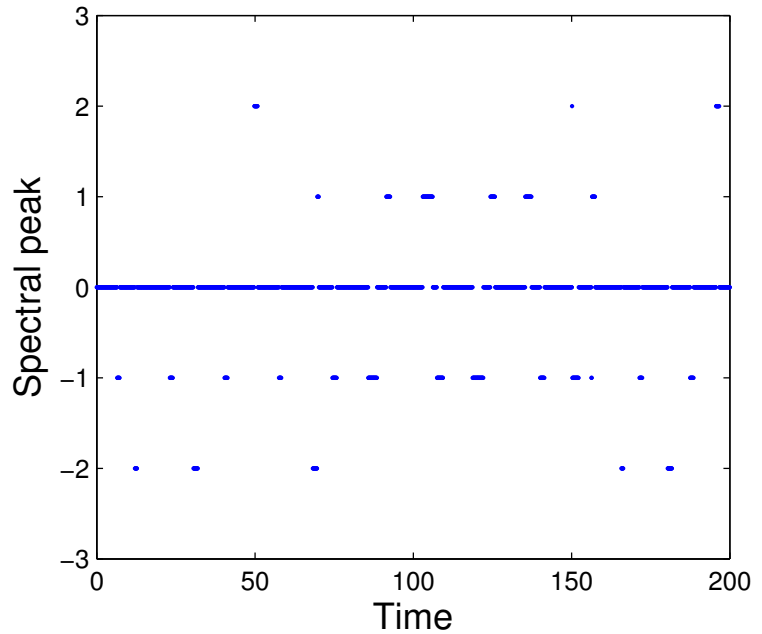

(d)

Figure 8: HONLS equation with $\epsilon=0.05, \Gamma=\beta=0$. Evolution of the (a) surface amplitude $|u(x, t)|$, (b) strength $S(t)$, (c) main Fourier modes, and (d) spectral peak using initial data (16) with $a=0.5, L=4 \sqrt{2} \pi, \delta=0.1$ for $0 \leq t \leq 200$.

A temporary downshift in the spectral peak occurs with each large wave. Figure 8(c) shows the time evolution of the main Fourier modes $\left|A_{k}(t)\right|$ for $k=0, \pm 1, \ldots, \pm 4$. During each of the modulation stages the zeroth mode loses energy as the upper and lower higher harmonics become excited. For the HONLS equation the total energy and the total energy flux are constant. This allows the energy to flow back to 
the zeroth mode and keeps the spectral center constant (in the numerical simulation the spectral center was conserved $\mathcal{O}\left(10^{-12}\right)$ ). The plot of $k_{\text {peak }}$ in Figure $8(\mathrm{~d})$ confirms that a permanent downshift does not occur, even when steep waves are encountered.

To compare the behavior of the waves for the HONLS $(\epsilon=0.05, \Gamma=\beta=0)$ and the NLS equations in the two UM regime, a set of numerical experiments was carried out by varying the amplitude and parameter $\tau$ in the two mode SPB initial data $U^{(2)}(x, 0 ;-5, \tau)$ with $L=4 \sqrt{2} \pi$. For each of the eleven values of $a$ used, $0.4 \leq a \leq 0.5$, twenty one $\tau$ values were chosen, $-9 \leq \tau \leq-4$, to include the effects of varying the time difference between the two modes in the SPB. For each value of $a$, the diagnostics are averaged over $\tau$. The exact two-mode SPBs (equation (15)) are evaluated at each $(x, t)$ to provide the NLS solutions.

At most two rogue waves occur in the SPB solution of the NLS equation depending on whether the modes are coalesced or not. In contrast, rogue waves generically emerge and occur intermittently throughout the entire HONLS time series. Figure 9 shows the (a) maximum strength and (b) lifetime of rogue waves obtained using two mode SPB initial data $U^{(2)}(x, 0 ;-5, \tau)$ for (i) the NLS equation (solid line) and (ii) the HONLS equation for short time, $0 \leq t \leq 40$, (dashed line) and long times, $0 \leq t \leq 400$, (dash-dot line).

On average, the maximum strength of the HONLS waves is larger than for the NLS waves as additional focusing occurs in the HONLS equation, as shown in Figure 9(a). The lifetime and number of rogue waves is greater in the HONLS equation than in the NLS (or the damped HONLS equations). In Figure 9(b) the difference in their respective lifetimes increases as the total time of the experiment increases. This is to be expected since rogue waves occur throughout the entire HONLS experiment. As a result, for a given set of initial data, the likelihood of obtaining rogue waves with the HONLS equation is greater than with the NLS equation.

\section{Rogue waves and temporary downshifting in the linear damped HONLS equation}

Equation (3) for the rate of change of the wave energy clearly shows that including linear damping by the wind $(\Gamma>0$ and $\beta=0)$ in the HONLS model results in a uniform damping of the individual Fourier modes (e.g. see Figure 10(c)). Permanent downshift is not expected for the linear damped HONLS equation since $k_{m}$ is constant in time. This is confirmed with both $k_{m}$ and $k_{\text {peak }}$ in the numerical experiments. Figure 10 shows the evolution of (a) the strength, (b) the spectral peak, and (c) the Fourier modes of the solution of (1) with $\epsilon=0.05, \Gamma=0.01, \beta=0$, using initial condition (16) with $a=.65, L=4 \sqrt{2} \pi$ and $\delta=0.1$. In Figure 10(b) $k_{\text {peak }}$ continues to return to the original dominant mode throughout the evolution. The spectral center is conserved within integrator accuracy, $\mathcal{O}\left(10^{-11}\right)$, of zero (not shown) and a permanent downshift does not occur.

Surprisingly, for short time evolutions, more rogue waves may appear in the damped HONLS equation than in the undamped HONLS equation. Figure 11 provides the wave strength $S(t)$ (solid line) and $|U|_{\max }(t)$ 


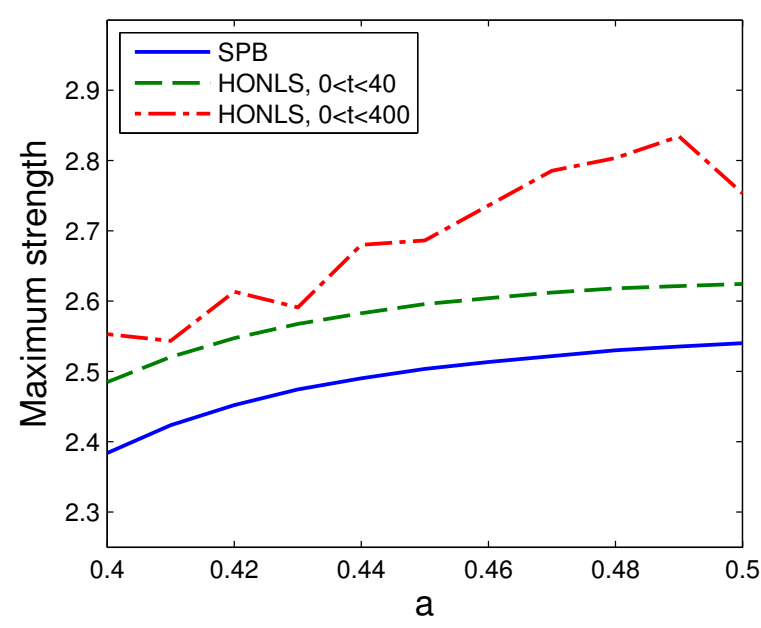

(a)

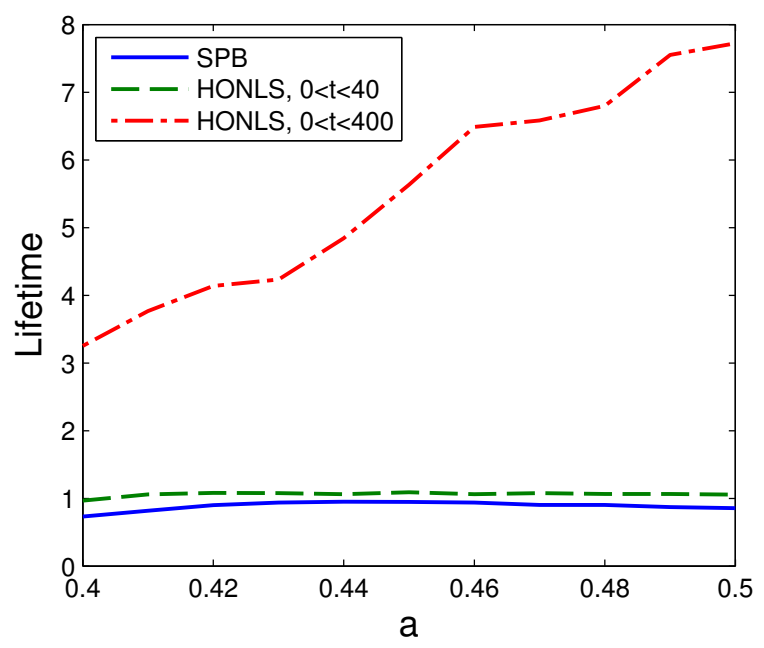

(b)

Figure 9: The (a) maximum strength and (b) lifetime of rogue waves obtained using two mode $\operatorname{SPB}$ initial data $U^{(2)}(x, 0 ;-5, \tau)$ for the NLS equation (solid line) and HONLS equation for short time, $0 \leq t \leq 40$, (dashed line) and long times, $0 \leq t \leq 400$, (dash-dot line).

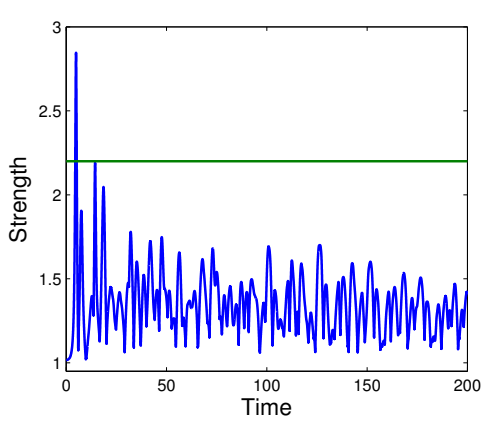

(a)

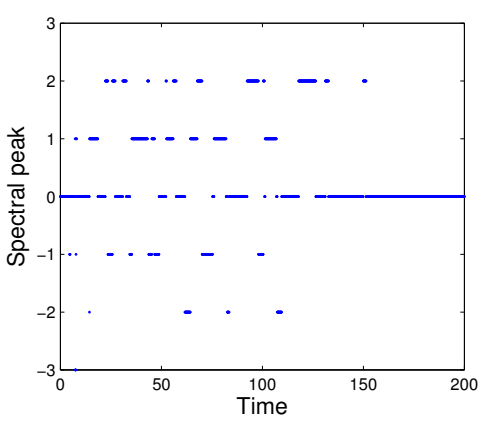

(b)

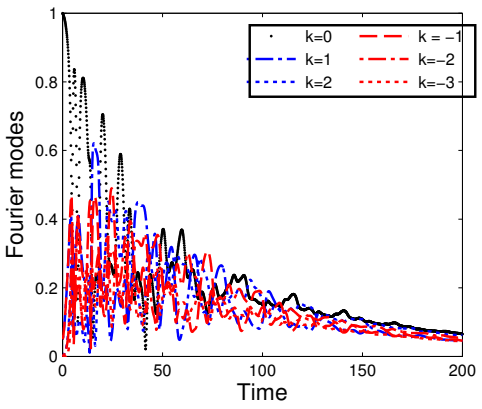

(c)

Figure 10: HONLS with linear damping, $\epsilon=0.05, \Gamma=0.01, \beta=0$. Evolution of (a) the strength, (b) the spectral peak, and (c) the Fourier modes using initial condition (16) with $a=.65, L=4 \sqrt{2} \pi$ and $\delta=0.1$.

(dotted line) for (a) the undamped HONLS equation $(\epsilon=0.05)$ and (b) the linear damped HONLS equation $(\epsilon=0.05, \Gamma=.005$, and $\beta=0)$ for initial condition (16) with $a=0.5, \delta=0.01$ and $L=4 \pi \sqrt{2}$. On this time frame the linear damped HONLS has two rogue waves while the undamped HONLS has only one. The damping alters the excitation time of the modes, allowing the modes to partially coalesce at $t \approx 30$ and produce the second rogue wave (Figure 11(b)). However, this is atypical and with damping the average strength and lifetime of rogue events decreases, which we subsequently address more fully.

In the linear damped HONLS experiments, $0 \leq t \leq 100$, we use initial data (16) with $\delta=0.1, L=4 \sqrt{2} \pi$, and where the amplitudes $a_{N}$ vary in the $N=1,2,3$ UM regimes initially, i.e. we let $.25 \leq a_{1} \leq .3, .45 \leq$ 


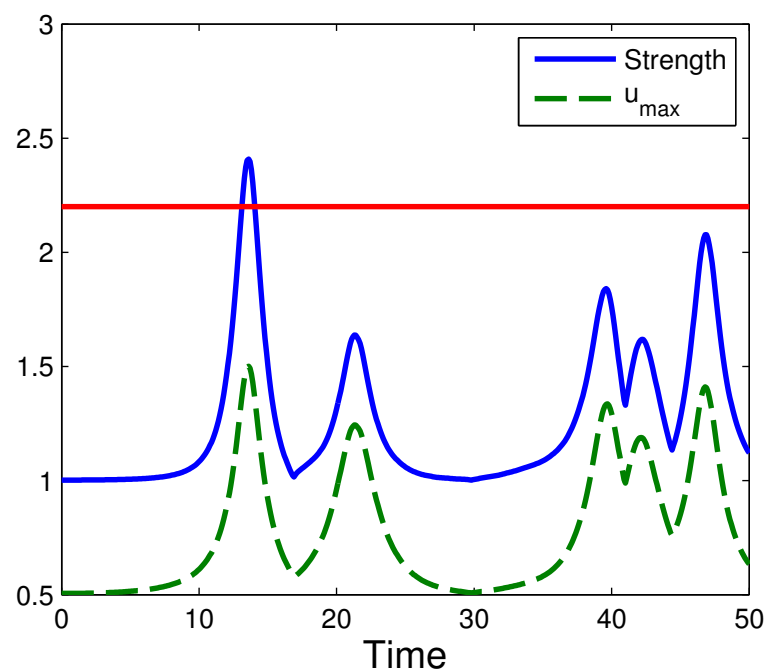

(a)

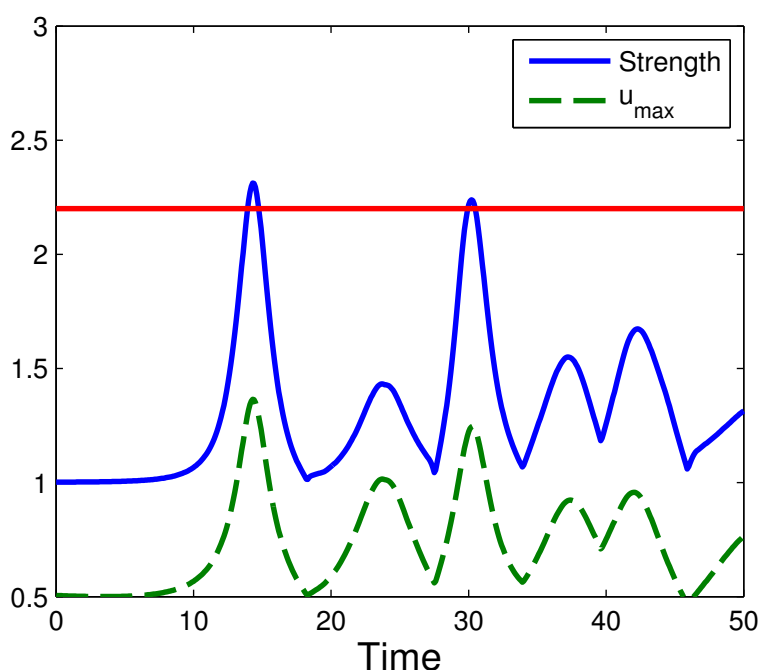

(b)

Figure 11: Strength $S(t)$ (solid line) and $|u|_{\max }$ (dashed line) for (a) HONLS Equation $\epsilon=0.05$; (b) HONLS Equation with Linear Damping, $\epsilon=0.05, \Gamma=0.005, \beta=0$, for IC $u_{0}=0.5(1+0.01 \cos \mu x)$, with $L=4 \sqrt{2} \pi$.

$a_{2} \leq .5$, and $.6 \leq a_{3} \leq .65$. The perturbation parameters for the evolution equation are $\epsilon=0.05, \beta=0$, and $0<\Gamma \leq 0.1$. For each value of $\Gamma=(0.002) j, j=0, \ldots, 50$, five numerical experiments were carried out by varying the amplitude $a_{N}$ in the initial condition for each of the three cases. For each value of $\Gamma$ the data for the strength and lifetime of the rogue waves was averaged over the five numerical experiments for each $N$.

Figure 12(a) shows that the lifetimes of rogue wave are generally decreasing in those experiments that have rogue waves in the undamped cases ( $N \geq 2$ UMs initially). For $\Gamma$ sufficiently large, rogue waves do not develop in the time series: this occurs at approximately $\Gamma=0.01$ when averaged over the experiments initially in the two UM regime and $\Gamma=0.03$ when averaged over the experiments initially in the three UM regime. Similarly, the number of rogue waves and the time of the last rogue wave are generally decreasing as linear damping is increased (not shown). Figure 12(b) shows the average maximum strength $S_{\max }(\Gamma)$ attained for $0 \leq t \leq 100$ which is perhaps more revealing. We find that when the wind strength $\Gamma$ is sufficiently small the sidebands can grow and rogue waves develop before the instability (and rogue waves) are eliminated in the time series. When $\Gamma$ is large, the modulational instability can be completely inhibited and the maximum strength of the waves decreases to the strength of the initial plane-wave.

\section{Rogue waves and permanent downshift in the nonlinear damped HONLS equation}

Nonlinear damping has quite a different effect on the Fourier spectrum than linear damping. Instead of being uniformly damped, equation (3) shows when $\beta>0$ and $\Gamma=0$, the individual Fourier modes are 


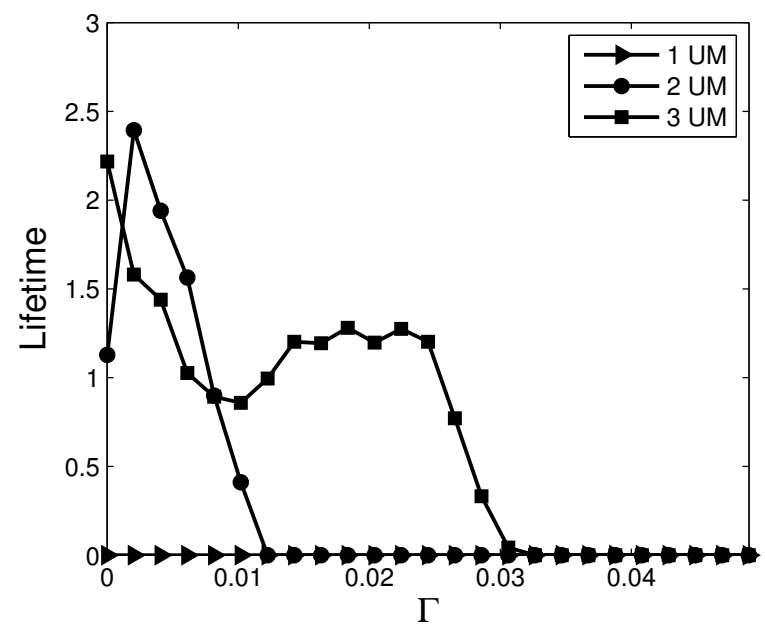

(a)

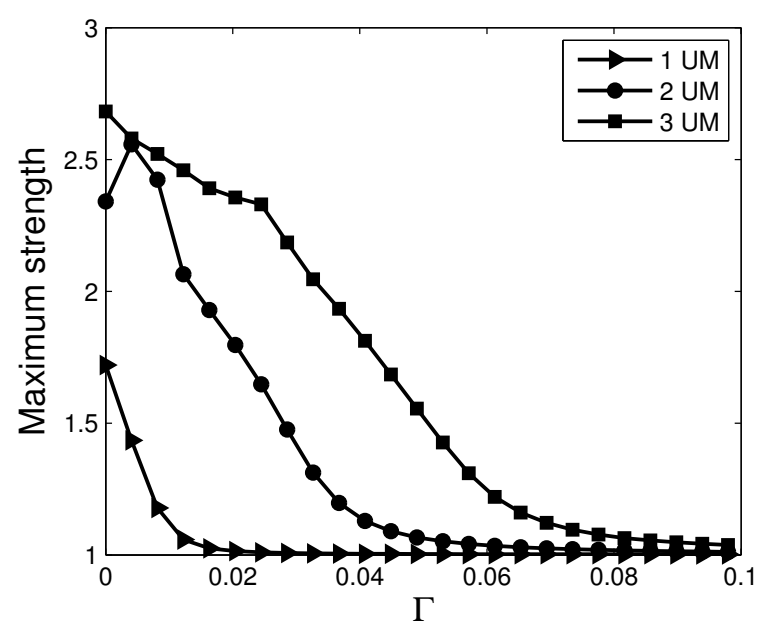

(b)

Figure 12: The (a) lifetime of rogue waves and the (b) maximum strength as a function of $\Gamma$ for the linear damped HONLS with $\epsilon=0.05, \beta=0$, and $0 \leq \Gamma \leq 0.1$ using the initial condition $u(x, 0)=a_{N}(1+0.1 \cos \mu x)$ and $0 \leq t \leq 100$.
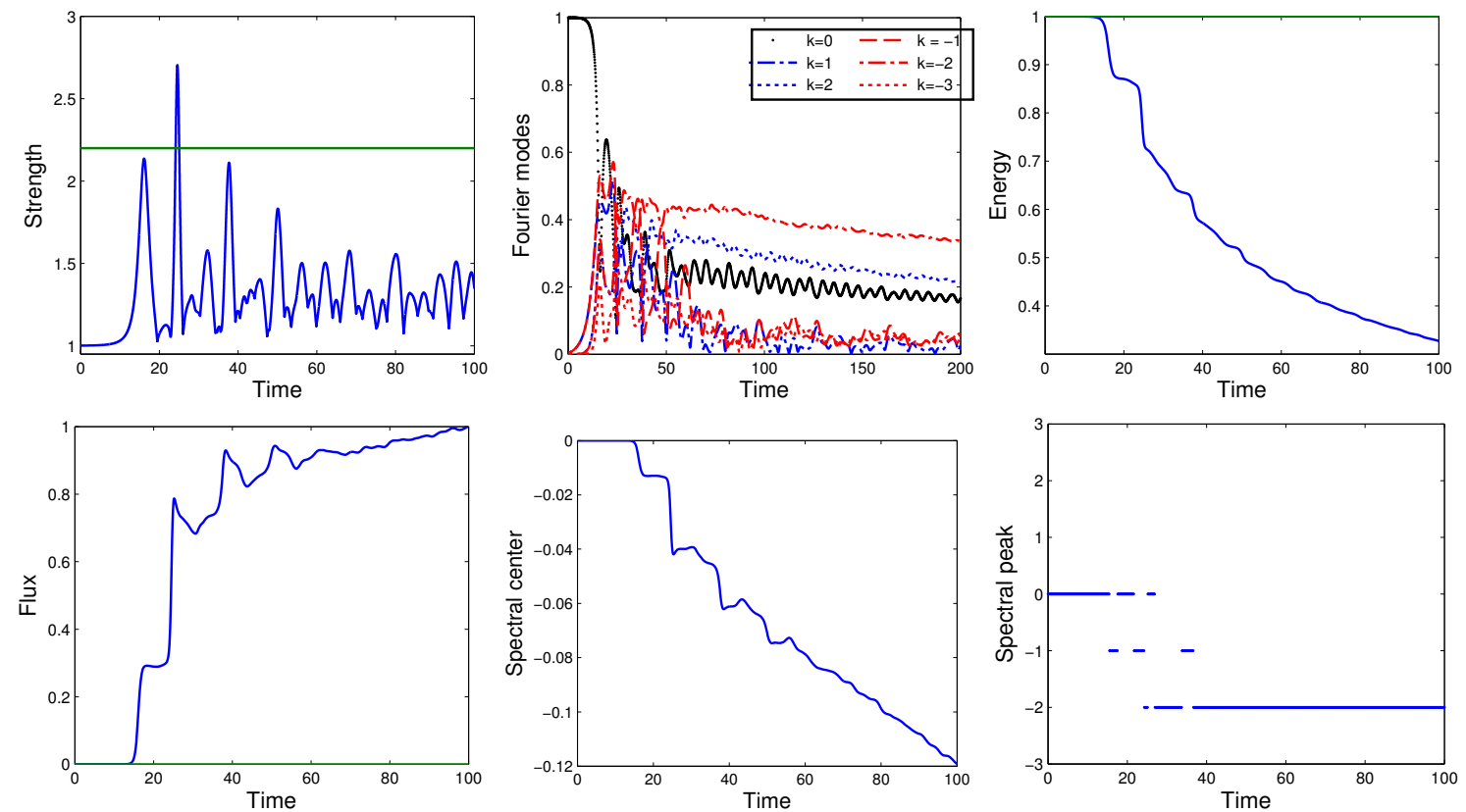

Figure 13: Nonlinear damped HONLS with $\epsilon=0.05, \beta=0.6, \Gamma=0$ using initial data (16) in the 2 UM regime with $a=0.45$, $\delta=0.01$ and $L=4 \sqrt{2} \pi$. The (a) strength $S(t)$, (b) Fourier modes, (c) energy, (d) momentum, (e) spectral center $k_{m}$, and (f) $k_{\text {peak }}$, for $0 \leq t \leq 100$. 


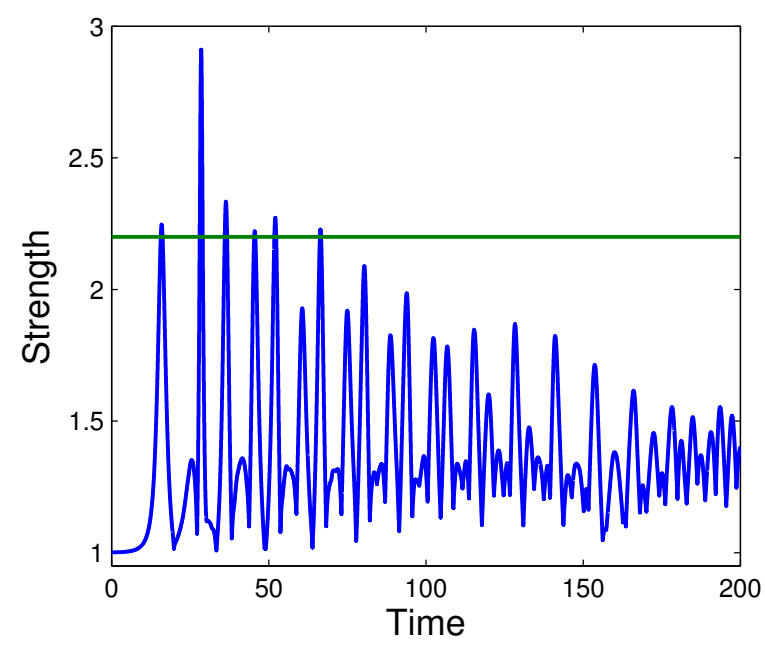

(a)

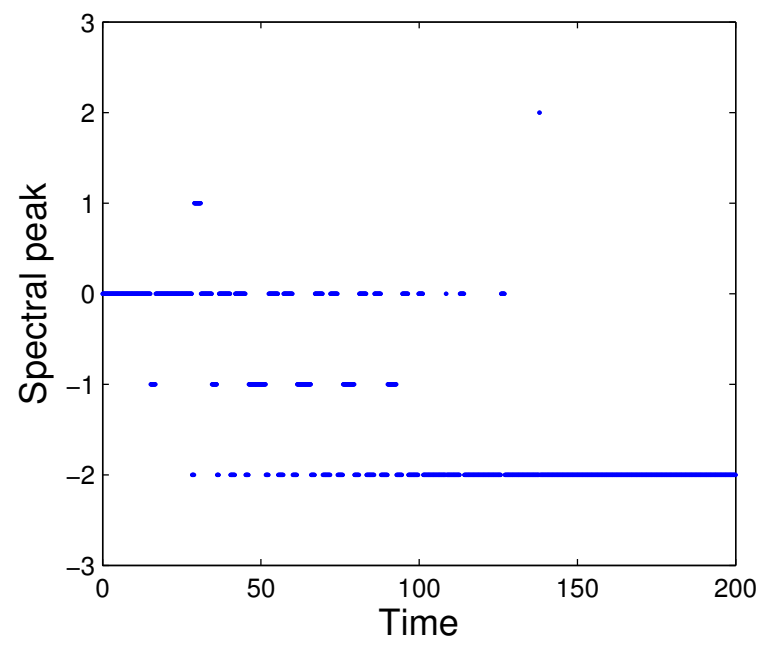

(b)

Figure 14: Nonlinear damped HONLS with $\epsilon=0.05, \beta=0.15, \Gamma=0$. The (a) strength and (b) the spectral peak $k_{p e a k}$ obtained using initial data (16) in the 2 UM regime with $a=0.45, \delta=0.01$, and $L=4 \sqrt{2} \pi$, for $0 \leq t \leq 200$.

damped at different rates. This nonlinear damping coupled with the Benjamin-Feir instability allows $k_{p e a k}$ to shift to a lower mode. To demonstrate the basic mechanism by which the $\beta$-term induces a permanent downshift in the spectrum, consider the following solution of equation (1) with $\epsilon=0.05, \beta=0.6$ and $\Gamma=0$, for initial data (16) with $a=0.45$ and $\delta=0.01,0 \leq t \leq 100$. The strength $S(t)$ and the main Fourier modes are shown in Figures 13(a-b).

In the numerical experiments, rapid, generally increasing/decreasing, step-like changes in the flux/energy with $|\dot{P}|>|\dot{E}|$ are essential for permanent downshift. The onset of downshifting occurs when the first large wave (albeit not a rogue wave) appears at $t \approx 16$, which produces an abrupt decay in the energy and growth in the flux as seen in Figure 13(c-d). This results in a rapid decrease in the spectral center $k_{m}$ and downward shift in the spectral peak, $k_{\text {peak }}$ at $t \approx 16$, Figures $13(\mathrm{e}-\mathrm{f})$. Afterwards, the energy and flux vary only slightly until the rogue wave is excited at $t \approx 27$. This triggers a further rapid decay in the energy and growth in the flux with the spectrum permanently downshifting from the carrier $k=0$ at $t \approx 25$. After permanent downshift, $k_{m}$ continues to decrease, but at a much slower rate. Figure 13(f) shows $k_{\text {peak }}$ continues to be active until the third large wave occurs at $t \approx 38$ at which time the spectrum settles to $k_{\text {peak }}^{\text {final }}=-2$. In the demodulation stage the beta term is small so that the energy and flux change at a much slower rate. Thus $k_{m}$ remains negative and does not upshift back.

The smaller the value of $\beta$, the slower the change in the energy and the flux, delaying a permanent downshift. As a case in point, Figure 14 shows (a) the strength and (b) the spectral peak of the solution of the nonlinear damped HONLS equation $(\epsilon=0.05, \beta=0.15)$ for the same initial data as in Figure 13. For $\beta=0.15$ the spectral peak, $k_{\text {peak }}$, permanently downshifts at $t \approx 127$, in contrast with the permanent 
downshift at $t \approx 27$ for $\beta=0.6$. Further, for $\beta=0.15$ the last rogue wave appears at $t \approx 67$, much later than for $\beta=0.6$.

To explore the effect of $\beta$ on downshifting and wave strengths (in particular for rogue waves), we use initial data (16) with $\delta=0.1$ in the (a) one UM regime $\left(L=2 \sqrt{2} \pi, 0.5 \leq a_{1} \leq 0.625\right)$ and the (b) two UM regime $\left(L=4 \sqrt{2} \pi, 0.4 \leq a_{2} \leq 0.48\right)$. The parameters for the evolution equation are $\epsilon=0.05, \Gamma=0$, and $0<\beta \leq 0.75$. For each of fourteen values of $\beta$, five experiments were conducted in both the one and two UM regimes by varying the amplitude $a_{N}$ in the initial condition.

Figure $15(\mathrm{a}-\mathrm{b})$ provides the time of permanent downshift $(\mathrm{x})$ and the time of the last rogue wave (square) averaged over the amplitudes in the (a) one UM regime and (b) two UM regime. The plots for a single amplitude $a$ are qualitatively the same. Significantly, in the set of experiments summarized in Figure 15 we find : 1) permanent downshift is observed in all the experiments for all $\beta>0.05$ and for all modulated unstable plane wave initial data ( $N \geq 1 \mathrm{UM}$ regimes). For the $N=1,2 \mathrm{UM}$ regime, the most unstable lower sideband becomes the dominant mode, i.e. $k_{\text {final }}^{\text {peak }}=-1,-2$ respectively. 2) The time of permanent downshift is a decreasing function of $\beta$, occurring rapidly for larger values of $\beta$. 3) Rogue waves typically do not develop after permanent downshift occurs. These results imply a downshifted sea-state does not allow for any further rogue waves.

Several comments are in order. First, although rogue waves do not occur in the one UM regime, the data in Figure 15(a) shows that the wave strengths are sufficient to trigger a permanent downshift for all $\beta$. Second, in $94 \%$ of the experiments in the two UM regime the last rogue wave occurs before permanent downshift (Figure 15(b)). In the remaining 4 experiments (6\%), at the time of permanent downshift the last rogue waves are already forming. That is, given the time required for the instability to saturate or grow to its maximum amplitude, $t_{S A T}$, for these four experiments $t_{L R W}-t_{P D S}<t_{S A T}$. This is a contrast to the results of the numerical experiments in the three UM regime, where the coalesced three mode solution triggered nonlinear damping sufficient to induce permanent downshift and eliminate rogue waves on the timeframe $0 \leq t \leq 200$ even for small $\beta<0.1$.

Third, Figure 16 shows the maximum strength obtained with the nonlinear damped HONLS $\epsilon=0.05$, $0 \leq \beta \leq 20, \Gamma=0$, using initial condition (16) with amplitudes for the $N=1,2,3$ UM regimes given by $a_{1}=0.25, a_{2}=0.45$, and $a_{3}=0.65$ and $\delta=0.1, L=4 \sqrt{2} \pi, 0 \leq t \leq 100$. Unlike the linear damped HONLS (Figure 12), nonlinear damping does not have as significant an effect on the initial stage of the modulational instability (see equation (9)). A large enough nonlinear damping can prevent rogue waves, but even for large $\beta$ the sidebands initially grow exponentially, although the growth rate $\sigma_{j}^{+}>0$ may be small, and the maximum wave strength remains greater than one. After the initial growth of the sidebands, the full nonlinear damping comes into effect along with the subsequent downshifting.

Finally, downshifting does not occur in the nonlinear damped HONLS equation for perturbations of stable plane waves. The changes in in $k_{m}$ are $\mathcal{O}\left(10^{-12}\right)$ and $k_{\text {peak }}$ did not fluctuate. A very small transfer 


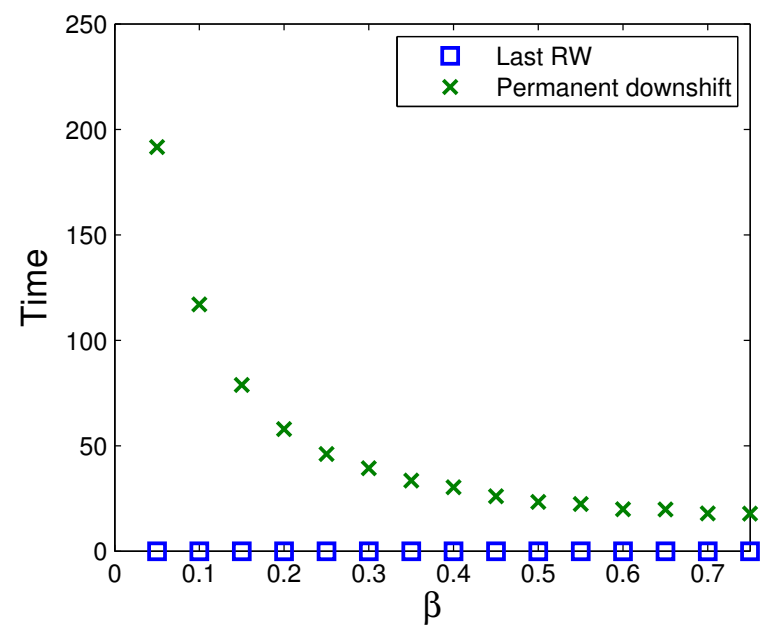

(a)

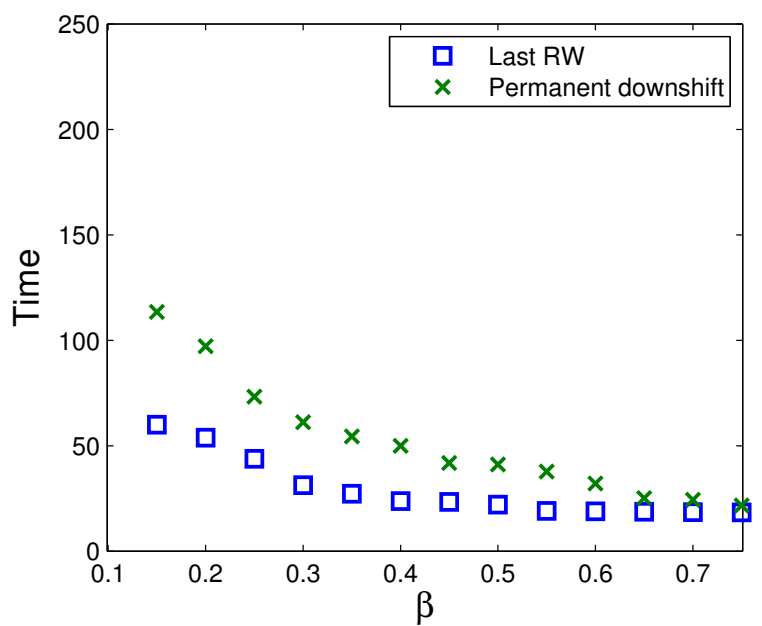

(b)

Figure 15: Nonlinear damped HONLS with $\epsilon=0.05,0<\beta<0.75, \Gamma=0$. For each $\beta$ the time of permanent downshift (x) and time of last rogue wave (square) averaged over 5 amplitudes in initial condition (16) in the (a) one UM regime $(L=2 \sqrt{2} \pi$, $0.5 \leq a \leq 0.625)$ and in the (b) two UM regime $(L=4 \sqrt{2} \pi, 0.4 \leq a \leq 0.48)$, for $0 \leq t \leq 250$.

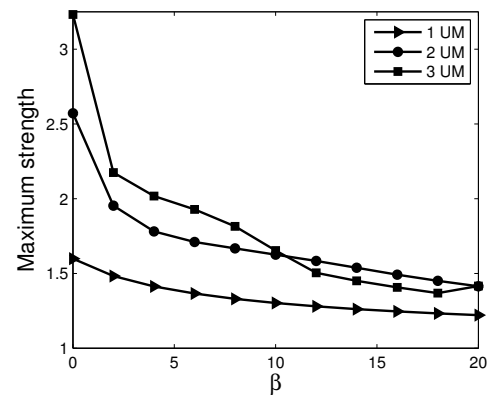

Figure 16: The maximum strength obtained with the nonlinear damped HONLS $\epsilon=0.05,0 \leq \beta \leq 20, \Gamma=0$, using initial condition (16) with amplitudes for the $N=1,2,3 \mathrm{UM}$ regimes given by $a_{1}=0.25, a_{2}=0.45$, and $a_{3}=0.65$ and $\delta=0.1$, $L=4 \sqrt{2} \pi, 0 \leq t \leq 100$.

of energy from the carrier to a lower mode occurs but there is no growth mechanism for this mode since the plane wave is stable and so downshifting does not occur.

\subsection{Characteristic features of the nonlinear damped evolution}

Features of the nonlinear damped evolution can be extracted from the previous set of experiments described in Figure 15. Figure 17(a) shows the effect of $\beta$ on the terminal spectral center, i.e. $k_{m}(200)$, for different amplitudes in the one UM regime. In the plots the solid curve is the average over the amplitudes. For each amplitude, $k_{m}(200)$ is a decreasing function of $\beta$. Further, for fixed $\beta$ as the amplitude of the initial condition increases the terminal spectral center $k_{m}$ decreases. Figure 17(b) shows for each initial 
amplitude in the one UM regime the maximum strength $\max _{t \in[0,200]} S(t)$ is a strictly decreasing function of $\beta$. Similarly, for fixed $\beta$, the maximum strength is a strictly decreasing function of the amplitude. This is in contrast to the behavior observed for $N \geq 2$ UMs (see Figure 18(b)) where due to a chaotic sea state averaged quantities must be examined.

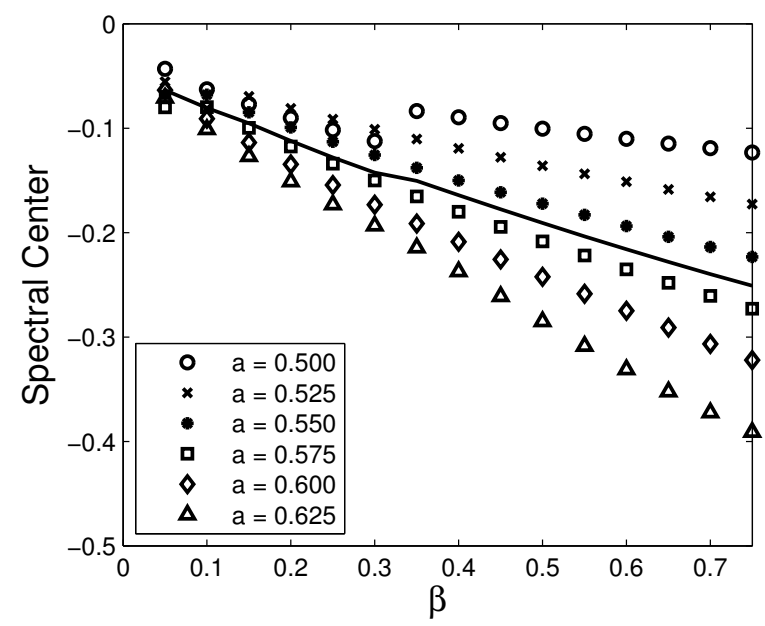

(a)

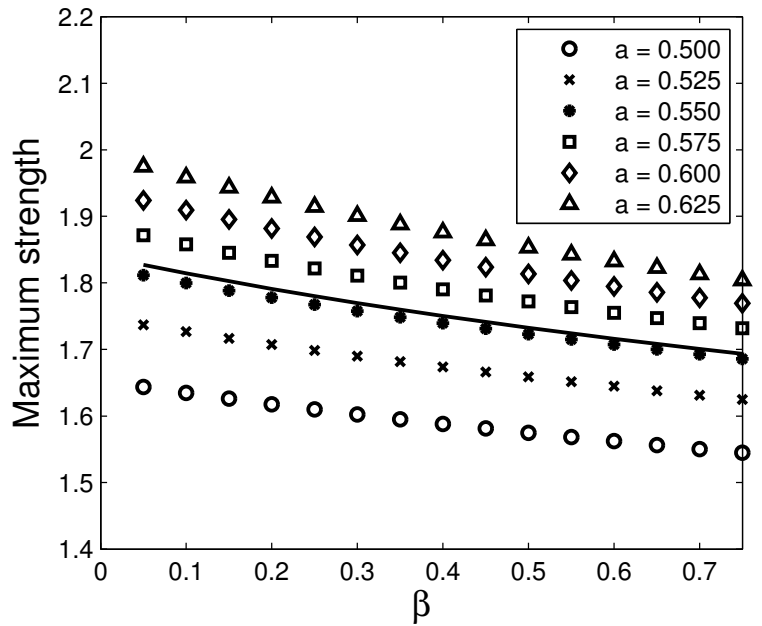

(b)

Figure 17: Nonlinear damped HONLS, $\epsilon=0.05,0 \leq \beta \leq .75, \Gamma=0$, for $0 \leq t \leq 400$. The (a) terminal spectral center and the (b) maximum strength obtained using initial data in the one UM regime with $L=2 \sqrt{2} \pi, \delta=0.1$, and specified amplitudes.

Figure 18 provides (a) the terminal spectral center $k_{m}$, (b) the maximum strength $\max _{t \in[0,200]} S(t)$, (c) the time of the last rogue wave, and (d) the lifetime of rogue waves for initial amplitudes in the two UM regime, $0.5<a<0.625$, for $0 \leq t \leq 200$. As in the one UM regime, Figure 18(a) shows that for fixed amplitudes in the two UM regime, $k_{m}(200)$ is a decreasing function of $\beta$, while for fixed $\beta, k_{m}(200)$ is a decreasing function of the amplitude.

In Figure 18(b) it is interesting to note that for two UMs the maximum strength actually increases for small $\beta$, which can also be seen in Figure 21. Although damping decreases the growth rates of the individual modes, one obtains larger waves for small $\beta$ due to changes in the focusing times and constructive interference. This behavior does not occur in the one UM mode regime as the possibility of coalescence doesn't exist. In the three UM regime [14], since the initial data was for a coalesced three mode rogue wave, the nonlinear damping split the coalesced state as well as damped it, resulting in significant decrease of the maximum strength. However, for the average over the amplitudes, there exists a critical $\beta^{*}$ for which the maximum strength decreases for $\beta>\beta^{*}$. For all values of the initial amplitude, the first rogue wave is only slightly delayed for all $\beta$ considered (not shown). Averaging the data over the amplitudes in the $N=2 \mathrm{UM}$ regime, Figures 18 (c-d) show as $\beta$ increases the time of the last rogue wave decreases to $T=20$ and the average lifetime (and number, not shown) of rogue waves decreases. 


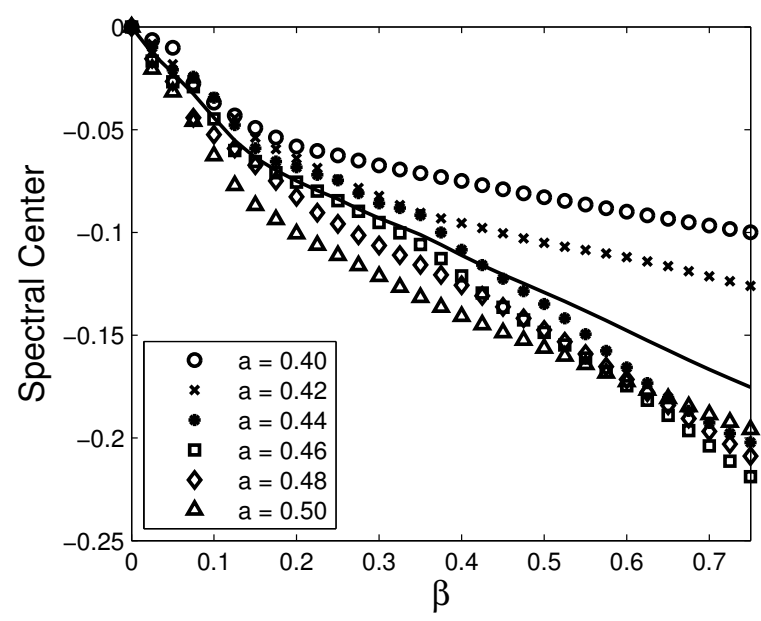

(a)

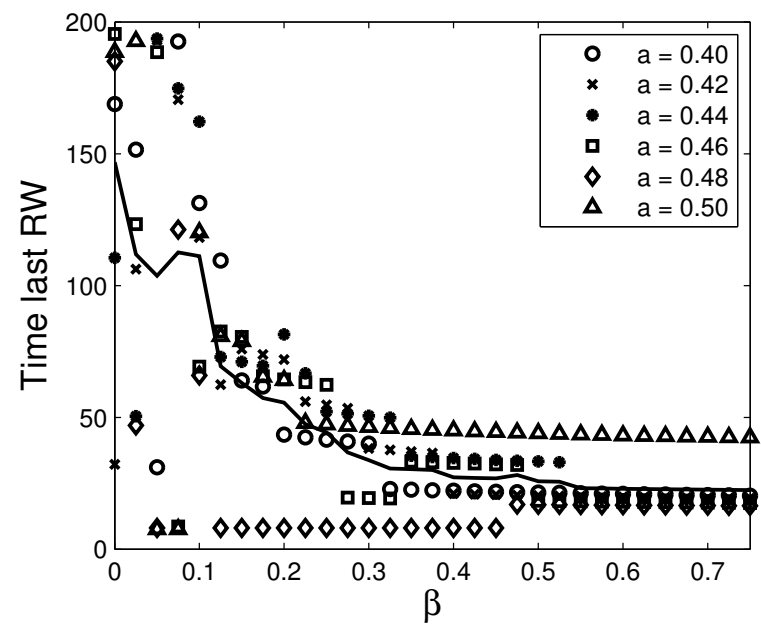

(c)

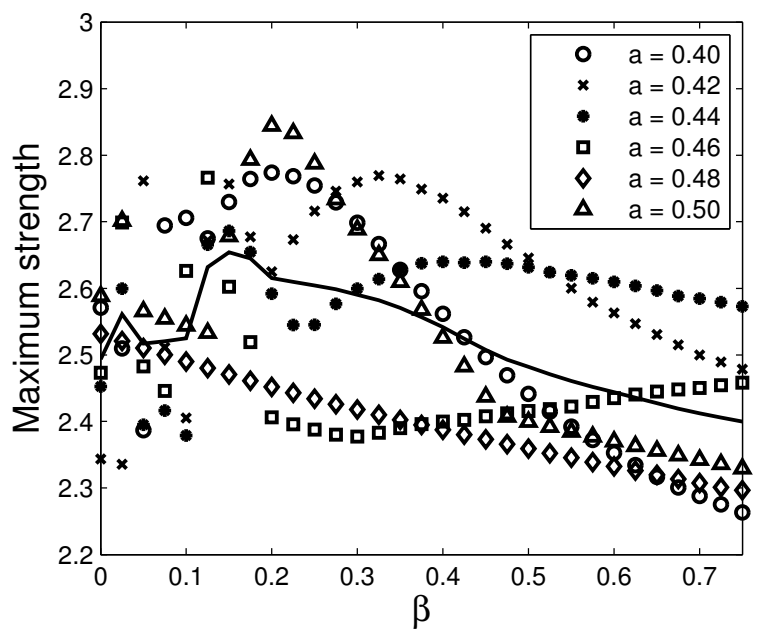

(b)

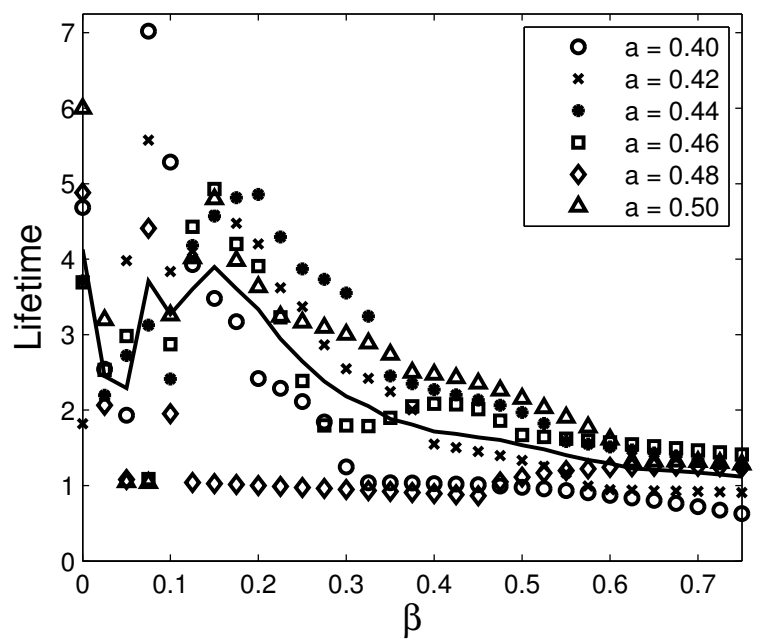

(d)

Figure 18: Nonlinear damped HONLS, $\epsilon=0.05,0 \leq \beta \leq .75, \Gamma=0$, for $0 \leq t \leq 200$. The (a) terminal spectral center, (b) maximum strength, (c) time of the last rogue wave, and (d) lifetime of rogue events obtained using specified amplitudes in the two UM regime with $L=4 \sqrt{2} \pi, \delta=0.1$.

\subsection{The effect of proximity to instabilities on rogue waves}

Currently there is considerable interest in developing criteria for predicting rogue waves $[13,2]$. In this section we examine how altering the proximity of the initial data to the plane wave and it's instabilities affects the characteristic features of the solution. Specifically, we show that as the initial data is chosen closer to the plane wave, the maximum strength of the waves as well as the number and lifetime of rogue waves increase on average while permanent downshift occurs sooner.

Figure 19 shows the (a) strength $S(t)$ and (b) spectral peak $k_{\text {peak }}(t)$ obtained with the nonlinear damped 
HONLS equation $\epsilon=0.05, \beta=.15, \Gamma=0,0 \leq t \leq 200$, for initial condition (16) with $a=0.45 \delta=0.0005$, $L=4 \sqrt{2} \pi$. Notice this experiment differs only in $\delta$, the perturbation of the plane wave, from the experiment shown in Figure 14 where $\delta=0.01$. Although for $\delta=0.0005$ spectral activity continues until $t \approx 110, k_{\text {peak }}$ permanently downshifts from the zeroth mode at $t \approx 90.2$ and the last rogue wave occurs at $t \approx 88$. In contrast, for $\delta=0.01 k_{\text {peak }}$ permanently downshifts from the zeroth mode at $t \approx 127$ and the last rogue wave occurs at $t \approx 66$. Further the maximum strength attained when $\delta=0.01$ is greater than when $\delta=0.1$.

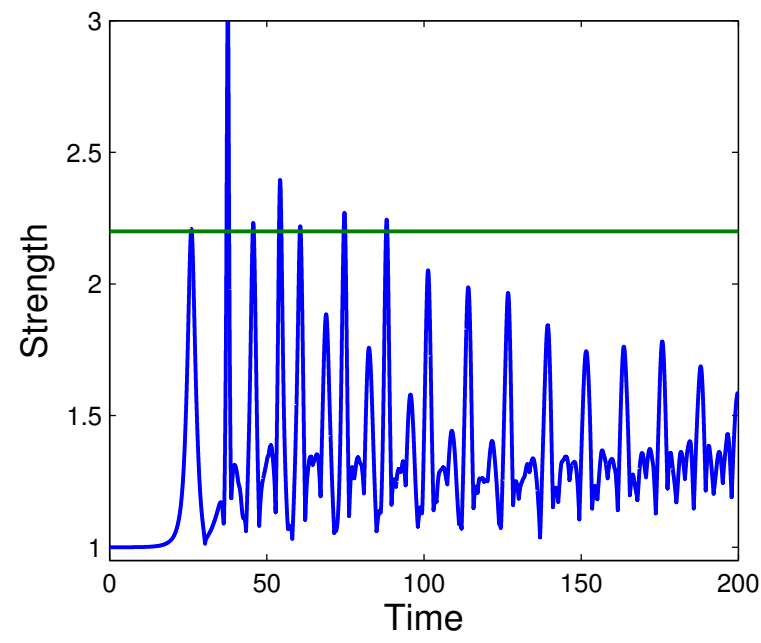

(a)

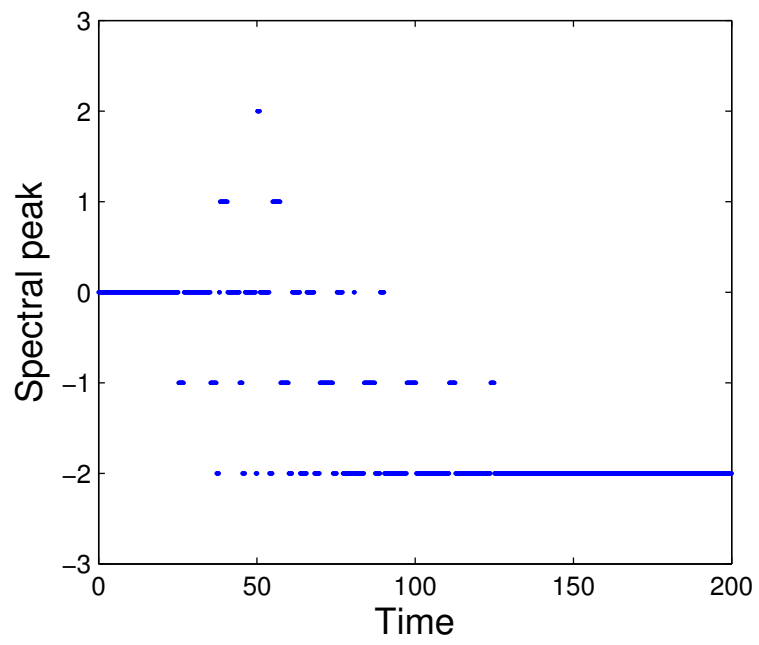

(b)

Figure 19: Nonlinear damped HONLS with $\epsilon=0.05, \beta=0.15, \Gamma=0$. Evolution of the (a) strength $S(t)$ and (b) spectral peak $k_{\text {peak }}$ for initial data (16) in the 2 UM regime with $a=0.45, \delta=0.0005$, and $L=4 \sqrt{2} \pi, 0 \leq t \leq 200$.

These observations suggest that the time of permanent downshift and the maximum strength of the wave potentially depend on the proximity to the unstable plane wave. To substantiate this, initial data for a set of experiments in the two UM regime is obtained by choosing fifteen values of the modulation parameter $\delta$ in the range $0.01 \leq \delta \leq 0.1$ for each of nine values of the amplitude in (16), $0.4 \leq a \leq 0.48, L=4 \sqrt{2} \pi$ The parameter $\hat{\delta}=a \delta$ measures the perturbation from the plane wave.

Figure 20(a-c) show how features of the nonlinear damped HONLS solution, for a fixed value of $\beta$ $(\epsilon=0.05, \beta=0.1, \Gamma=0)$ depend on the proximity to the plane wave. The result of a single experiment is displayed as a data point. The solid line represents the average obtained over 15 uniform bins in the $\hat{\delta}$ range of the (a) lifetime, (b) maximum strength, (c) number of rogue waves, and (d) terminal spectral center. We find that there is a $\hat{\delta}^{*}$ such that for $\hat{\delta}>\hat{\delta}^{*}$ as $\hat{\delta}$ increases, the lifetime, maximum strength and number of rogue waves decrease while the spectral center increases (i.e. the downshift from zero decreases) as $\hat{\delta}$ increases. Additionally, the time of the last rogue wave and the time of the maximum strength is slightly delayed (not shown).

Figure 21 presents the dependence of the maximum strength on both $\beta$ as well as $\delta$ for solutions of the 
nonlinear damped HONLS, $\epsilon=0.05,0 \leq \beta \leq 0.75, \Gamma=0,0 \leq t \leq 200$, obtained for initial data (16) in the two UM regime with (i) $\delta=0.1$ (circle), (ii) $\delta=0.01$ (square), (iii) $\delta=0.001$ (triangle). Here the data point represents the average over five amplitudes $0.40 \leq a \leq 0.48$ for each $\delta$. The solid curve connects the data points to improve visual clarity. For each $\beta$, as $\delta$ decreases, i.e. as the initial data gets closer to the plane wave and it's instabilities, the maximum strength increases. As in Figure 18(b), for each $\delta$, there exists a critical $\beta^{*}$ for which the maximum strength decreases for $\beta>\beta^{*}$.

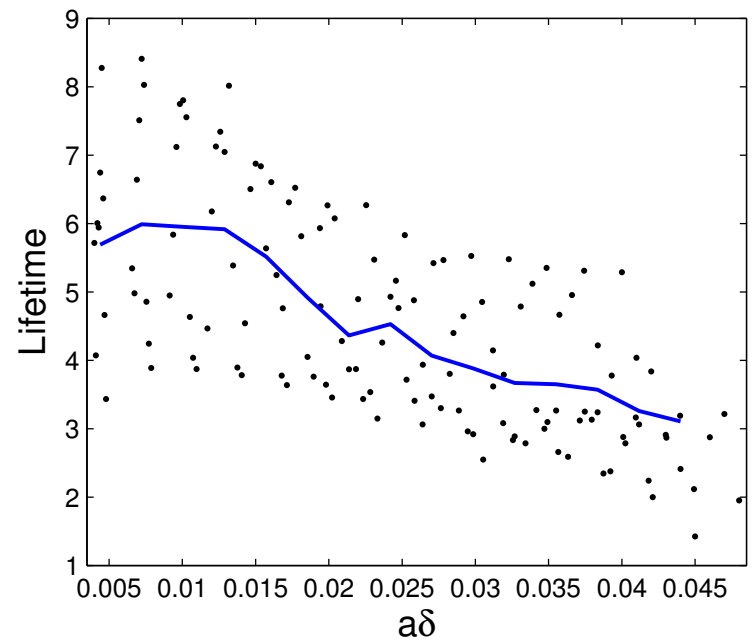

(a)

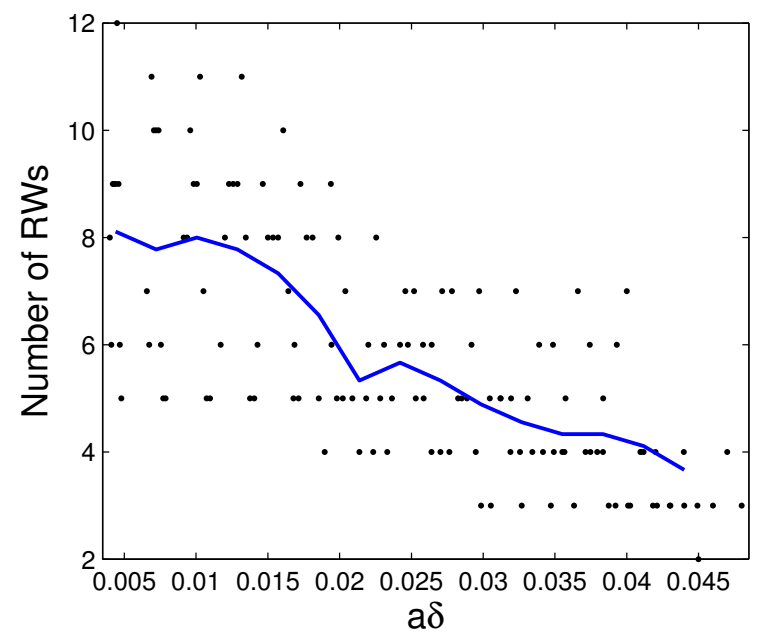

(c)

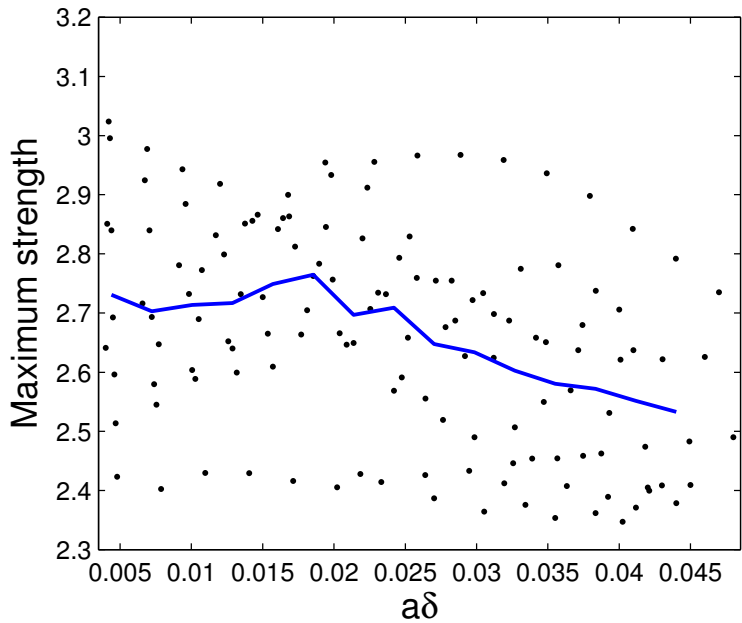

(b)

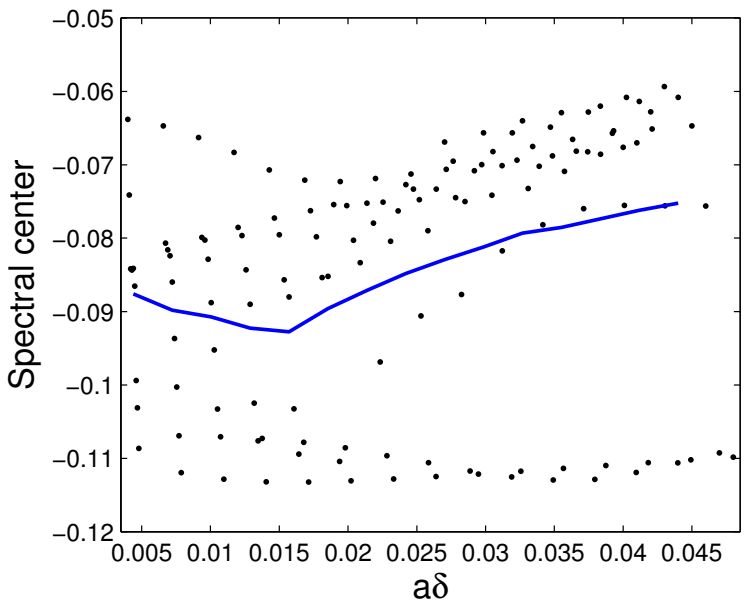

(d)

Figure 20: Nonlinear damped HONLS, $\epsilon=0.05, \beta=.1$, and $\Gamma=0$, for $0 \leq t \leq 600$. The amplitude and modulation parameter $\delta$ in initial condition (16) are varied in the two UM regime. The solid line represents the average of the (a) lifetime, (b) maximum strength, (c) number of rogue waves, and (d) terminal spectral center. 


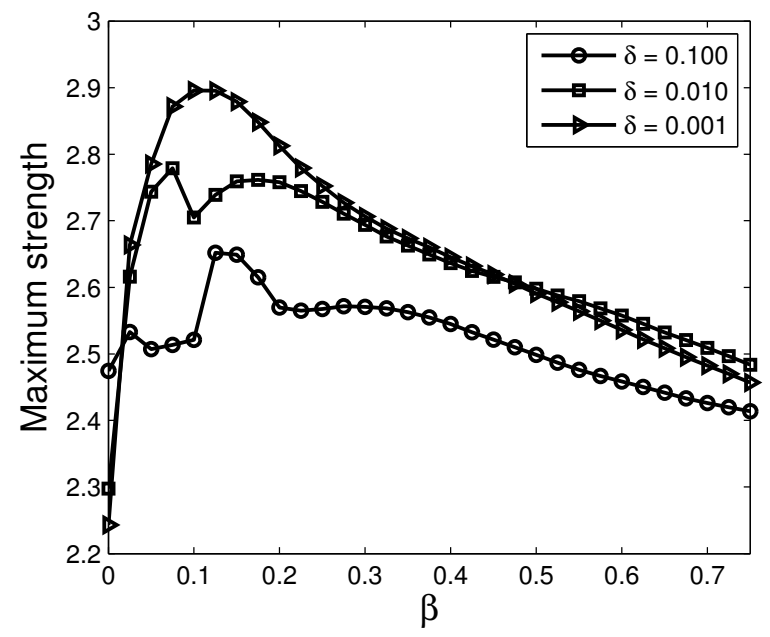

Figure 21: Two UM initial condition in nonlinear damped HONLS, $\epsilon=0.05,0 \leq \beta \leq .75, \Gamma=0$, for $0 \leq t \leq 200$. The maximum strength obtained for (i) $\delta=.1$, (ii) $\delta=.01$, (iii) $\delta=.001$. The solid line is the average over the amplitudes for a given $\delta$.

\subsection{The effect of wave strength on the time of permanent downshift}

Recall Figure 19 and Figure 14 provide an example of two experiments, differing only by their distance to the plane wave (as measure by $\delta$ ), which yielded different maximum strengths. Further, the solution with the larger maximum strength downshifted more quickly. This raises the question of how the time of permanent downshift depends upon the wave strength and rogue wave activity.

To address this question a set of experiments was carried out using the nonlinear damped HONLS equation. Nine values of the amplitude in initial condition (16) are chosen in each of the one and two UM regimes, i.e. for $0.3 \leq a \leq 0.38,0.4 \leq a \leq 0.48$, respectively, with $L=4 \sqrt{2} \pi$. For each amplitude nine values of the modulation parameter $\delta$ are chosen, $10^{-3} \leq \delta \leq 10^{-1}$. Figure 22 provides the time of permanent downshift for the nonlinear damped HONLS, $\epsilon=0.05, \Gamma=0$, and $\beta=0.1$ (circle), $\beta=0.2$ (square), or $\beta=0.4$ (triangle), as a function of the average maximum strength in (a) the one UM regime and in (b) the two UM regime. Each data point represents the average computed by grouping the data over 10 uniform bins (the curve is for visual clarity). For the smaller values of $\beta$ the average time of permanent downshift decreases as the maximum strength of the wave increases in both the one and two UM regimes. As $\beta$ increases, the dependence of the time of permanent downshift on the strength diminishes. For example, in the two UM regime when $\beta=0.4$ the time of permanent downshift is almost uniform for $S_{\max }>2.4$.

Relating this to the results in subsection 5.3 on the relation between the perturbation from the plane wave in the initial condition, we find that as $\delta$ decreases the maximum strength increases and subsequently the length of time it takes for the solution to permanently downshift decreases. Restated, larger $\delta$ values in the initial condition take longer to downshift, and smaller $\delta$ values (which are closer to the plane wave) 
downshift sooner.

Returning to our Conjecture on the essentials for permanent downshift in our numerical experiments, the initial data which supports the conjecture in a very clear way are initial data chosen closer to the plane wave in the two or three UM regime so as to quickly excite steep rogue waves.

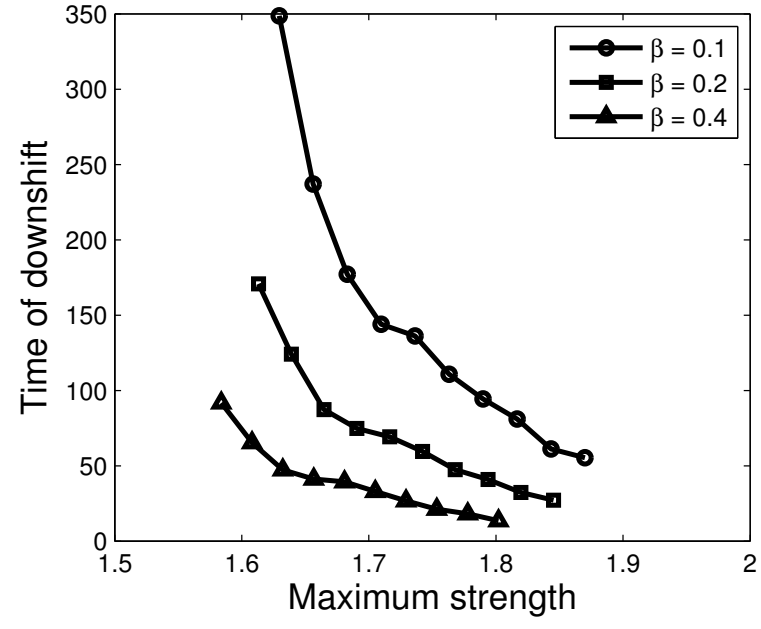

(a)

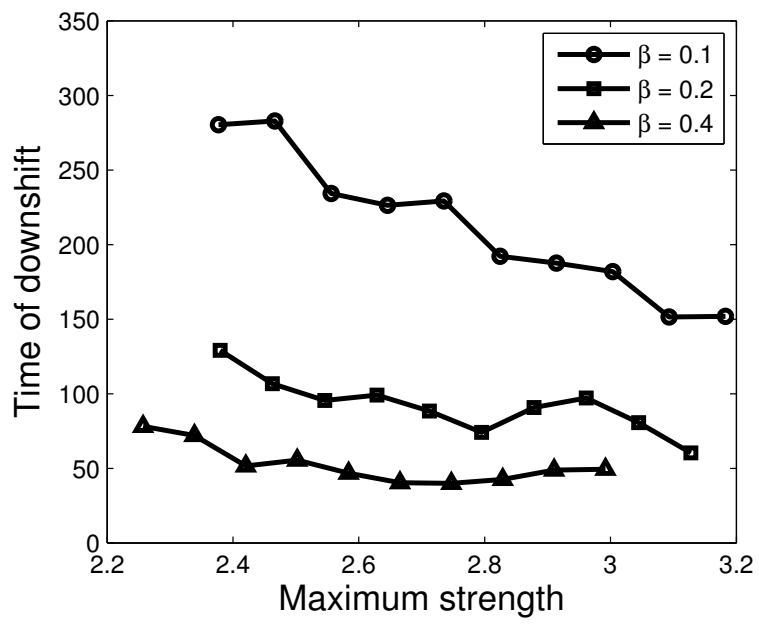

(b)

Figure 22: Nonlinear damped HONLS, $\epsilon=0.05, \Gamma=0$, and $\beta=.1$ (circle), $\beta=.2$ (square), or $\beta=.4$ (triangle) for $0 \leq t \leq 450$ with experiments required to downshift by $t=400$. The time of permanent downshift as a function of the maximum strength for initial condition (16) in the (a) one UM regime, $0.3 \leq a \leq 0.38$ and (b) two UM regime, $0.4 \leq a \leq 0.48$.

\section{Permanent downshift and rogue waves in a wind-driven and nonlinear damped sea}

In this section we explore the combined effects of wind and nonlinear damping on the HONLS evolution. For simplicity, when $\beta>0$ and $\Gamma \neq 0$, equation (1) is referred to as the perturbed HONLS equation. In the numerical experiments the initial data is of the form (16), with $\delta=0.1$ and an appropriate amplitude and period for each of the $N=1,2,3 \mathrm{UM}$ regimes. For each initial condition, seventy-five experiments are carried out by selecting $\beta=0.1, \ldots, 0.5$, where fifteen values of $\Gamma$ are selected for each value of $\beta$. Further, $\Gamma$ is considered active for differing lengths of time $\left(T_{\text {wind }}\right)$ as specified in the figure labels. The ranges in $\Gamma$, chosen to ensure stability of the forced experiments, depend on the number of nearby unstable modes. For each value of $T_{\text {wind }}$, the curves presented are the averages over the five $\beta$ values considered.

When nonlinear damping dominates, the mechanism for permanent downshift in the perturbed HONLS equation is qualitatively similar to that described in Section 5, e.g. see Figure 13, for the nonlinear damped HONLS. In general, permanent downshift takes longer to occur in the perturbed HONLS experiments than in the nonlinear damped HONLS experiments. For larger wind strength relative to the strength of the nonlinear damping, there is a higher percentage of experiments which do not downshift on the time frame 
under study and a higher percentage of experiments with spectral activity in the sidebands after the time of permanent downshift (similar to the multistage downshifts in Figure 19) and some cases of upshifting when $\Gamma<0$. For a different physical scenario, i.e. when growth by the wind and wave breaking are considered [27], if the wind is sufficiently strong the tendency to downshift disappears. Our results on the combined effect of forcing by the wind and nonlinear damping on permanent downshift are consistent with this study in terms of the diminishing percentage of experiments that downshift.

Figure 23 and Figure 24 show the terminal spectral center, $k_{m}(200)$, and the maximum strength, respectively, obtained using the perturbed HONLS equation $(\epsilon=0.05,0.1 \leq \beta \leq 0.5,-0.006 \leq \Gamma \leq 0.006$, averaged over $\beta$ ) for various $T_{\text {wind }}, 0 \leq t \leq 200$, for initial condition (16), $L=4 \sqrt{2} \pi$, in (a) the one UM regime, $a=0.3$, (b) the two UM regime, $a=0.48$, and (c) the three UM regime, $a=0.67$.

Figure 23 shows for a given $T_{\text {wind }}$ the terminal spectral center is an increasing function of $\Gamma$ (each data point represents the average over five $\beta$ values). In other words, forcing by the wind causes the downshift (from zero) to decrease further than when wind is not present whereas damping by the wind diminishes the downshift. Comparing the terminal spectral center obtained in Figure 23 (a), (b), and (c), one finds this behavior is qualitatively similar when there are $N \geq 2 \mathrm{UMs}$ present. Moreover, for a given $\Gamma$, as $T_{\text {wind }}$ increases, the terminal spectral center decreases more when $\Gamma<0$ (forcing), while the terminal spectral decreases less when $\Gamma>0$ (damping). Thus the inclusion of damping by the wind and the duration of $T_{\text {wind }}$ serve to inhibit the downshift due to nonlinear damping, while forcing by the wind and the duration of $T_{\text {wind }}$ enhance the downshifting effects of the nonlinear damping.

Recall downshifting in this model is due to nonlinear damping which has the property that steeper waves produce stronger damping (and downshifting). One can qualitatively explain the results as follows: linear damping reduces the strength and peakedness of the waves, which in turn decreases the nonlinear damping and downshift from zero. Linear forcing has the opposite effect. This behavior is nearly identical across the different values of $\beta$ considered.

In the one UM regime, Figure 24(a) shows that for each $T_{\text {wind }}$ the maximum strength is a decreasing, approximately linear, function of $\Gamma$. This behavior is also seen for individual $\beta$ values (not shown). In the one UM regime there are no rogue waves in the HONLS evolution. Similarly, for the values of $\beta, \Gamma$, and $T_{\text {wind }}$ considered in the perturbed HONLS evolution, there are no rogue waves in the one UM regime.

With the inclusion of wind, one could expect that features of the rogue events such as strength, number of events, lifetime would increase with linear forcing and decrease with linear damping. However, for the small values of $\Gamma$ considered, this is not always the case. In the two UM regime, the wind changes the focusing time of the modes and allows for constructive interference of the modes. When averaged over $\beta$, the maximum strength of the rogue waves are generally decreasing across the $\Gamma$ range examined in the two and three UM regimes as seen in Figures 24(b-c). Similarly, the number of rogue waves is a decreasing function of $\Gamma$ for each $\beta$ in the two and three UM regimes (not shown). The time of the last rogue wave is 


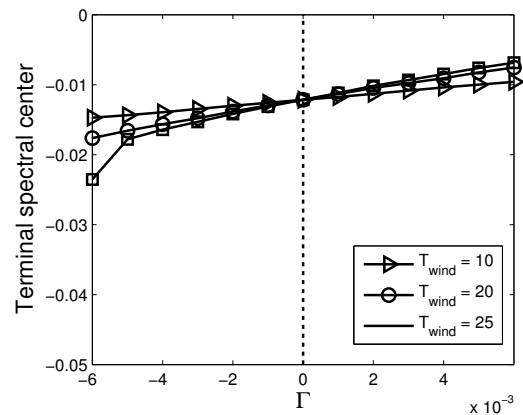

(a)

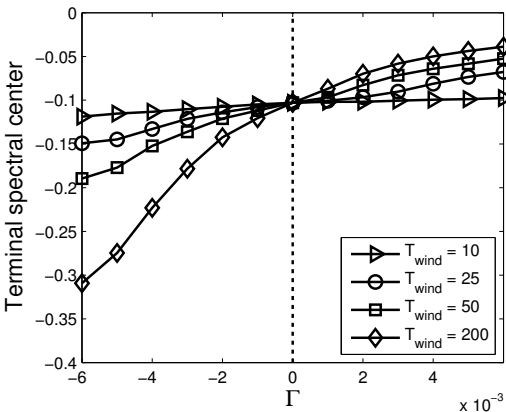

(b)

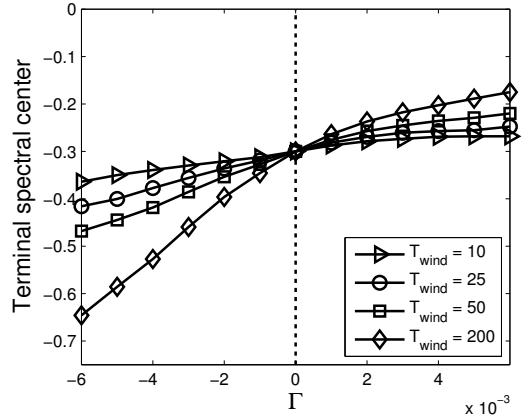

(c)

Figure 23: Perturbed HONLS with $\Gamma$ applied for $0 \leq t \leq T_{\text {wind }}$ averaged over five $\beta$ values using initial condition (16) with $\delta=0.1, L=4 \sqrt{2} \pi, \epsilon=0.05$ for $0 \leq t \leq 200$. The terminal spectral center for (a) one UM $(a=0.30)$, (b) two UMs $(a=.48)$ and $(\mathrm{c})$ three UMs $(a=.67)$. [65 experiments]

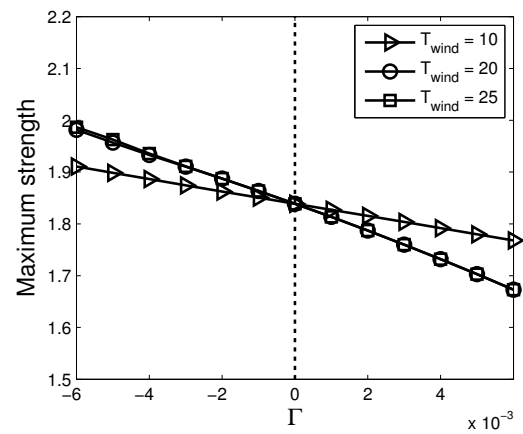

(a)

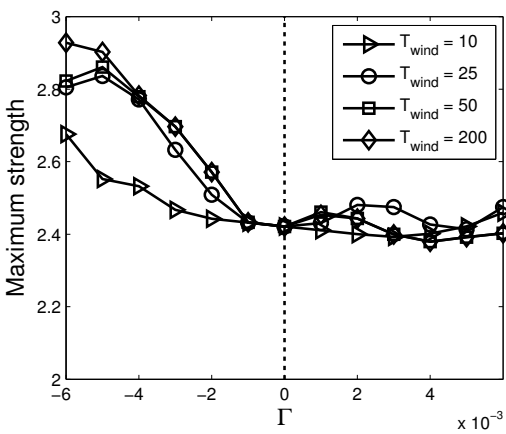

(b)

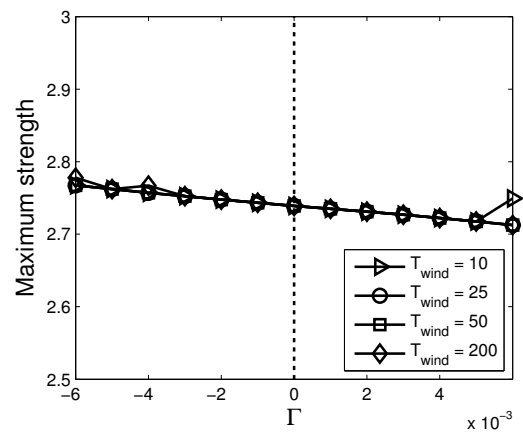

(c)

Figure 24: Perturbed HONLS with $\Gamma$ applied for $0 \leq t \leq T_{\text {wind }}$ averaged over five $\beta$ values using initial condition (16) with $\delta=0.1, L=4 \sqrt{2} \pi, \epsilon=0.05$ for $0 \leq t \leq 200$. The maximum strength for (a) one UM $(a=0.30)$, (b) two UMs $(a=.48)$ and (c) three UMs $(a=.67)$. [65 experiments $]$

much later for small values of $\beta$ when linear forcing is included (not shown). For large values of $\beta$ however, the magnitude of linear forcing in the range considered had little impact on the time of the last rogue wave.

\subsection{Time of permanent downshift and time of the last rogue wave}

It was shown in section 5 that permanent downshift occurs $\forall \beta>0.05$ when $\Gamma=0$ (for $N \geq 1 \mathrm{UMs}$ ) and that rogue waves do not occur after permanent downshift in the two and three UM regimes. Here we examine this issue when both nonlinear damping and wind are present. Figure 25 presents the time of permanent downshift (x) and the time of the last rogue wave (square) obtained using the perturbed HONLS equation $\epsilon=0.05, \Gamma= \pm 0.005, T_{\text {wind }}=50,0.15 \leq \beta \leq 0.75$ for $0 \leq t \leq 200$. (Linear damping delays downshifting beyond $t=200$ in most of the experiments when $\beta \leq 0.1$ so these values of $\beta$ are not included.) Thirteen values of $\beta$ are chosen in the range $.15 \leq \beta \leq .75$. For each $(\Gamma, \beta)$ pair, five values of the initial amplitude 
in initial condition (16) are used in the numerical experiments in (a) the two UM regime $(.4 \leq a \leq .48)$ and (b) the three UM regime $(.57 \leq a \leq .65)$. For the experiments which downshifted by $t=200$, the times of permanent downshift are averaged over the relevant amplitudes. The plots for a single amplitude $a$ are qualitatively the same.

With damping, $\Gamma=.005$, permanent downshift occurs in $94 \%$ of the numerical experiments in the two and three UM regimes. In $95 \%$ of these downshifted experiments there are no rogue waves after permanent downshift. In the anomalous experiments, although the last rogue wave occurs after the last time the spectral peak returned to the zeroth mode, the last rogue wave occurred before spectral activity in the sidebands

ceased, i.e. before $k_{\text {peak }}^{\text {final }}$ is achieved. Figures 25 (a)and (c) show these results averaged over the different initial amplitudes in the experiment set. As $\beta$ increases, the time of permanent downshift and the time of the last rogue wave move closer.

When the waves are forced, $\Gamma=-.005$, permanent downshift is delayed and occurs on the time frame in question $0 \leq t \leq 200$ in $70 \%$ and $97 \%$ of the numerical experiments in the two UM and three UM regimes, respectively, primarily for the larger values of $\beta$. In $96 \%$ of the downshifted experiments, rogue waves do not occur after permanent downshift. In the outlier experiments, either the rogue wave was already forming at the time of permanent downshift or activity in the lower sidebands had not ceased. The experiments in the two and three UM regimes are summarized in Figure 25(b) and Figure 25(d) respectively. As in the nonlinear damped HONLS evolution, with the combined effects of wind and nonlinear damping we again find that typically rogue waves do not occur in a downshifted sea. The primary differences is that fewer experiments experience permanent downshift.

\section{Conclusions}

In Section 2.4 we presented a linear stability analysis of Stokes waves for the new nonlinear damped HONLS, and the setup of the numerical experiments. Determining permanent downshift is not always straightforward and the diagnostics were presented in Section 2.3, along with a conjecture on criteria for permanent downshift. We introduced the asymptotic peak wavenumber as also relevant when relating the time of the last rogue wave with a settling of spectral activity. We clarified an overlooked feature of the one mode SPB solution, $U^{(1)}(x, t ; \rho)$, of the NLS equation (see equation $\left.(13)\right)$. Specifically, when $U^{(1)}(x, t ; \rho)$ is over a Stokes wave with one UM it does not satisfy the strength definition of a rogue wave. The strength of $U^{(1)}(x, t ; \rho)$ increases with $L$, allowing it to qualify as a rogue wave for larger $L$. However, the one UM regime is typified by waves with smaller strength and steepness. Further rogue waves do not appear in the HONLS evolution in the one UM regime. On the other hand, we showed the family of two mode SPBs always has members which are rogue waves.

Damping by the wind was examined in Section 4 where we showed that the inclusion of linear damping 


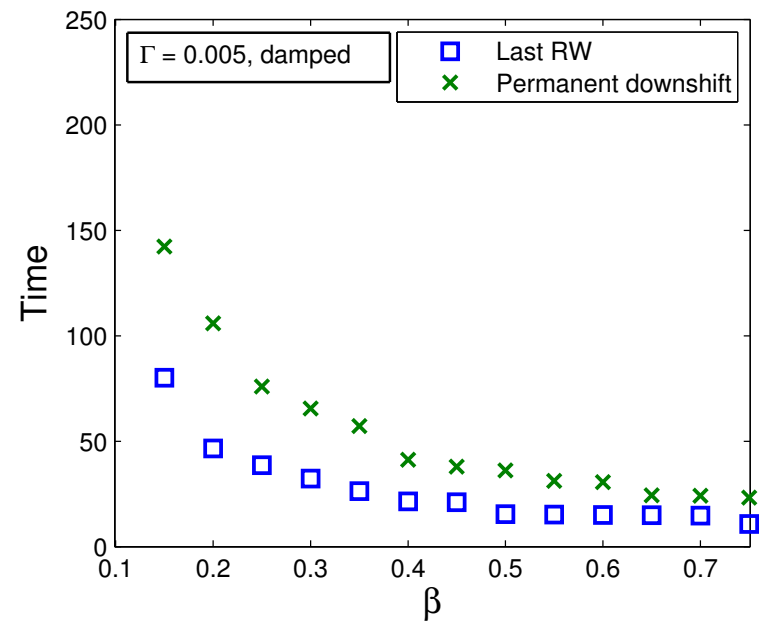

(a)

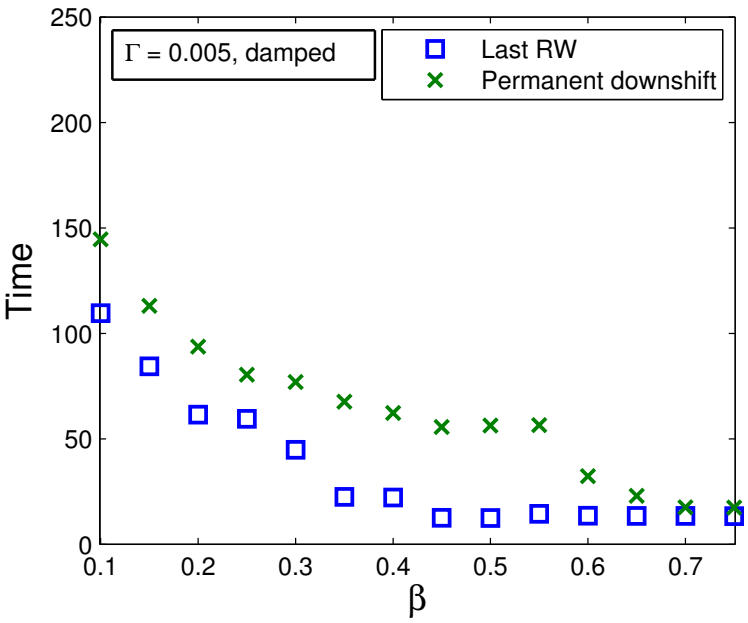

(c)

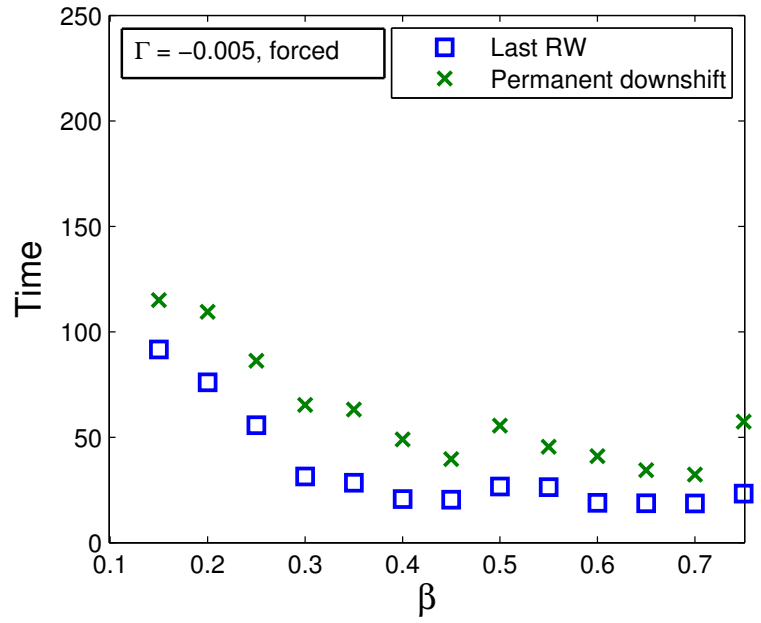

(b)

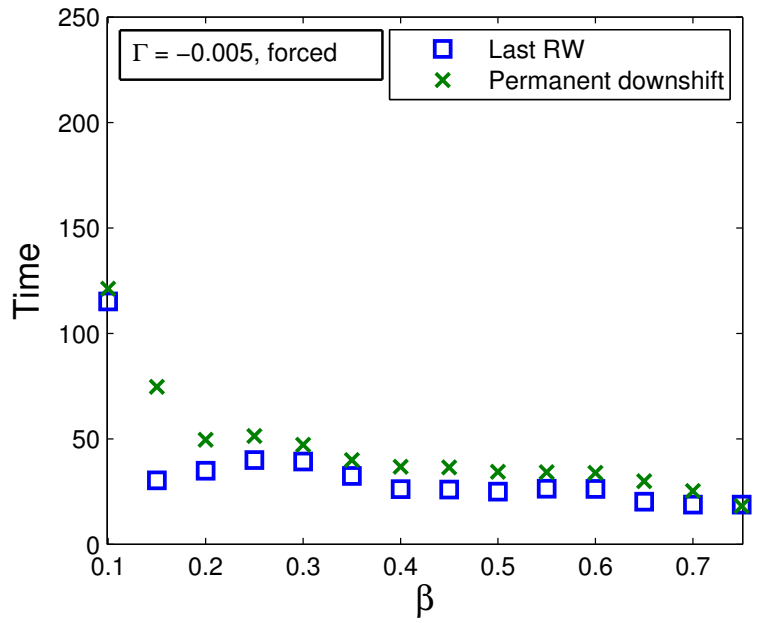

(d)

Figure 25: The perturbed HONLS equation $\epsilon=0.05,0<\beta<0.75$, and $\Gamma= \pm 0.005$. The time of permanent downshift (x) and time of last rogue wave (square) (a) for $\Gamma=0.005$ and (b) for $\Gamma=-0.005$ averaged over 5 amplitudes in the two UM regime, and (c) for $\Gamma=0.005$ and (d) for $\Gamma=-0.005$ averaged over 5 amplitudes in the three UM regime. 
typically leads to smaller maximum strengths, fewer rogue waves and shorter lifetimes. Consistent with the study on stabilizing the BF instability [26], we found that when the wind strength is sufficiently small the sidebands can grow and rogue waves develop before the instability (and rogue waves) are eliminated. When the wind strength is large, the modulational instability can be completely inhibited and the maximum strength of the waves decreases to the strength of the initial plane-wave.

Rogue waves and permanent downshift in the presence of nonlinear damping were investigated in Section 5. In our experiments, rapid, generally increasing/decreasing, step-like changes in the flux/energy with $|\dot{P}|>|\dot{E}|$ were shown to be essential for permanent downshift. For the $N=1,2$ UM regimes, in the nonlinear damped HONLS evolution we found : 1) Permanent downshift is observed for all $\beta>0.1$ for all modulated Stokes wave initial data, 2) The time of permanent downshift is a decreasing function of $\beta$, occurring rapidly for larger values of $\beta$. 3) As $\beta$ increases the average maximum strength, lifetime and time of the last rogue wave decrease. 4) Typically rogue waves do not develop after permanent downshift. These results imply a downshifted sea-state does not allow for any further rogue waves.

In contrast with the linear damped HONLS, nonlinear damping does not have as significant an effect on the initial stage of the modulational instability (see equation (9)). A large enough nonlinear damping can prevent rogue waves but even for large $\beta$ the sidebands initially grow exponentially, although the growth rate may be small. After the initial growth of the sidebands, the full nonlinear damping comes into effect along with the subsequent downshifting.

We found that the proximity of the initial data to unstable plane waves impacts the characteristic features of the rogue waves in the nonlinear damped HONLS evolution. In Section 5.2 we showed that as the initial data gets closer to the plane wave, the maximum strength, number, and lifetime of rogue waves increase while the time of permanent downshift decreases. From the alternate perspective of how the wave strength and rogue wave activity affect the time of permanent downshift, in section 5.3 we showed that the greater the wave strength, the more rogue waves, or the longer their lifetime, the earlier permanent downshift occurs.

In Section 6 we explored the combined effects of nonlinear damping and wind (the perturbed HONLS equation (1) with $\beta>0$ and $\Gamma \neq 0$ ) on rogue waves and permanent downshift. We demonstrated that the inclusion of forcing by the wind leads to larger maximum strengths and lifetime of rogue waves even when including nonlinear damping, whereas damping by the wind causes the rogue waves to have lower maximum strength and shorter lifetimes, on average. When nonlinear damping dominates, the mechanism for permanent downshift in the perturbed HONLS equation is qualitatively similar to that for the nonlinear damped HONLS, although permanent downshift takes longer to occur in the perturbed HONLS experiments. For large wind strength relative to the strength of the nonlinear damping, there is a higher percentage of experiments which do not downshift on the time frame under study and a higher percentage of experiments with spectral activity in the sidebands after the time of permanent downshift. For a different physical scenario, i.e. when growth by the wind and wave breaking are considered [27], if the wind is sufficiently 
strong the tendency to downshift disappears. Our results on the combined effect of forcing by the wind and nonlinear damping on permanent downshift are consistent with this study in terms of the diminishing percentage of experiments which downshift.

We found that for a given $\beta$, the frequency downshift (from zero) decreases as linear damping by the wind $\Gamma$ increases. On the other hand, when wind forcing is present, the frequency downshift (from zero) increases as the magnitude of the linear forcing increases. In other words, we found that with wind damping, nearly all the experiments had permanent downshift although the spectral center downshifts less. When wind forcing is included, fewer experiments downshifted on the time frame examined, but in the cases that downshifted, the spectral center downshifts more. In the experiments on the combined effects of wind and nonlinear damping we found that typically rogue waves do not occur after permanent downshift.

\section{Acknowledgments}

The authors gratefully acknowledge the support of the National Science Foundation, Grant DMS-1108973.

\section{References}

[1] N. Akhmediev, A. Ankiewicz, M. Taki, Waves that appear from nowhere and disappear without a trace, Phys. Lett. A 373 (2009) 675-678

[2] A. Ankiewicz, P.A. Clarkson, N. Akhmediev, Rogue waves, rational solutions, the patterns of their zeros and integrable relations, J. Phys. 43 (2010) 9

[3] A. Calini, C.M. Schober, Homoclinic chaos increases the likelihood of rogue waves, Phys. Lett. A 298 (2002) 335-349

[4] A. Calini, C.M. Schober, Observable and reproducible rogue waves, J. Opt. 15 (2013) 105201

[5] J.D. Carter, C. Contreras, Stability of plane wave solutions of a dissipative generalization of the nonlinear Schrödinger equation, PhysicaD 237 (2008) 3292-3296

[6] S.M. Cox, P.C. Matthews, Exponential time differencing for stiff systems. J. Comput. Phys. 176 (2002) 430-455

[7] K. Dysthe, Note on a modification to the nonlinear Schrodinger equation for deep water, Proc. Roy. Soc. London A 369 (1979) 105-114

[8] K. Dysthe, K. Trulsen, H. Krogstad, H. Socquet-Juglard, Evolution of a narrow band spectrum of random surface gravity waves, J. Fluid Mech. 478 (2003) 1-10

[9] O. Gramstad, K. Trulsen, Hamiltonian form of the modified nonlinear Schrdinger equation for gravity waves on arbitrary depth, J. Fluid Mech. 670 (2011) 404-426

[10] T. Hara, C.C. Mei, Frequency downshift in narrow-banded surface waves under the influence of wind, J. Fluid Mech. 230 (1991) 429-477

[11] M. Hederi, A. Islas, K. Reger, C.M. Schober, Efficiency of exponential time differencing schemes for nonlinear Schrodinger equations, Math. Comput. Simul. (2013)

[12] D.M. Henderson, H. Segur, The role of dissipation in the evolution of ocean swell, J. Geophys. Res. Oceans 118 (2013) $5074-5091$

[13] A. Islas, C. Schober, Predicting rogue waves in random oceanic sea states, Phys. of Fluids 17 (2005) 031701-1:4

[14] A. Islas, C. Schober, Rogue waves, dissipation, and downshifting, PhysicaD 240 (2011) 1041-1054 
[15] Y. Kato, M. Oikawa, Wave number downshift in Modulated Wavetrain through a Nonlinear Damping effect, J. Phys. Soc. Japan 64 (1995) 4660-4669

[16] A.Q.M. Khaliq, J. Martin-Vaquero, B.A. Wade, M. Yousuf, Smoothing Schemes for Reaction-Diffusion Systems with Nonsmooth Data. J. Comp. App. Math. 223 (2009) 374-386

[17] C Kharif, R. Kraenkel, M. Manna, and R Thomas, The modulational instability in deep water under the action of wind and dissipation. J. Fluid Mech., 664:138149, 2010.

[18] B. Lake, H. Yuen, W. Ferguson, Envelope solitons and recurrence in nonlinear deep water waves: theory and experiment, Rocky Mountain J. Math. 8 (1978) 105-116

[19] B. Lake, H. Yuen, H. Rungaldier, W. Ferguson, Nonlinear deep-water waves: theory and experiment. Part 2. Evolution of a continuous wave train, J. Fluid Mech. 83 (1977) 49-74

[20] A. Leblanc, Amplification of nonlinear surface waves by wind. Phys. of Fluids, 19(10):101705, 2007.

[21] E. Lo, C.C. Mei, A numerical study of water wave modulation based on a higher order nonlinear Schrödinger equation, J Fluid Mech. 150 (1985) 395-416

[22] D.W. McLaughlin, C.M. Schober, Chaotic and Homoclinic behavior for numerical discretizations of the nonlinear Schrodinger equation, PhysicaD 57 (1992) 447-465

[23] N. Mori, T. Yasuda, H. Mase, T. Tom, Y. Oku, Projection of extreme wave climate change under global warming, Hydrol. Res. Lett. 4 (2010) 15-19

[24] A. Osborne, M. Onorato, M. Serio, The nonlinear dynamics of rogue waves and holes in deep-water gravity wave trains, Phys. Lett. A 275 (2000) 386

[25] D.H. Sattinger, V.D. Zurkowski, Gauge theory of Bäcklund transformations, PhysicaD 26 (1987) 225

[26] H. Segur, D. Henderson, J. Carter, J. Hammack, C. Li,D. Pheiff, K. Socha, Stabilizing the Benjamin-Feir instability, J. Fluid Mech. 539 (2005) 229-271

[27] K. Trulsen, K. Dysthe, Action of wind stress and breaking on the evolution of a wave train. In M. L. Banner, R.H.J. Grimshaw (Eds.), Breaking Waves, Springer Verlag, 1992, pp. 243-249.

[28] K. Trulsen, K. Dysthe, Frequency downshift in three-dimensional wave trains in a deep basin, J. Fluid Mech. 352 (1997) 359-373.

[29] Y. Uchiyama, T. Kawahara, A possible mechanism for frequency downshift in nonlinear wave modulation, Wave Motion 20 (1994) 99-110 